\title{
The ESCRT-III isoforms CHMP2A and CHMP2B display different effects on membranes upon polymerization
}

Maryam Alqabandi ${ }^{1 \dagger}$, Nicola de Franceschi ${ }^{1 \dagger}$, Sourav Maity ${ }^{2}$, Nolwenn Miguet ${ }^{3}$, Marta Bally ${ }^{4}$, Wouter H. Roos ${ }^{2}$, Winfried Weissenhorn ${ }^{3 \dagger}$, Patricia Bassereau ${ }^{1+}$ and Stéphanie Mangenot ${ }^{1 *+}$ (i)

\begin{abstract}
Background: ESCRT-III proteins are involved in many membrane remodeling processes including multivesicular body biogenesis as first discovered in yeast. In humans, ESCRT-III CHMP2 exists as two isoforms, CHMP2A and CHMP2B, but their physical characteristics have not been compared yet.

Results: Here, we use a combination of techniques on biomimetic systems and purified proteins to study their affinity and effects on membranes. We establish that CHMP2B binding is enhanced in the presence of PI( 4,5$) \mathrm{P} 2$ lipids. In contrast, CHMP2A does not display lipid specificity and requires CHMP3 for binding significantly to membranes. On the micrometer scale and at moderate bulk concentrations, CHMP2B forms a reticular structure on membranes whereas CHMP2A (+CHMP3) binds homogeneously. Thus, CHMP2A and CHMP2B unexpectedly induce different mechanical effects to membranes: CHMP2B strongly rigidifies them while CHMP2A (+CHMP3) has no significant effect.
\end{abstract}

Conclusions: We therefore conclude that $\mathrm{CHMP2B}$ and $\mathrm{CHMP} 2 \mathrm{~A}$ exhibit different mechanical properties and might thus contribute differently to the diverse ESCRT-III-catalyzed membrane remodeling processes.

Keywords: Endosomal sorting complexes Required for Transport (ESCRT), Reconstituted system, Bottom up approach, Lipid-protein interactions, Membrane, Mechanical properties, Giant unilamellar vesicles (GUV), Micropipette, Atomic force microscopy (AFM)

\section{Background}

The endosomal sorting complex required for transport (ESCRT-III) complex is involved in a variety of cellular processes [1] such as biogenesis of multivesicular bodies (MVB) [2], plasma membrane wound repair [3], neuron pruning [4], dendritic spine formation [5], nuclear envelope repair or nuclear envelope sealing during

\footnotetext{
* Correspondence: stephanie.mangenot@curie.fr

${ }^{+}$Maryam Alqabandi and Nicola de Franceschi are co-first authors.

†Patricia Bassereau, Winfried Weissenhorn, and Stéphanie Mangenot are cosenior authors.

'Laboratoire Physico Chimie Curie, Institut Curie, Université PSL, Sorbonne Université, CNRS UMR168, 75005 Paris, France

Full list of author information is available at the end of the article
}

telophase [6,7], abscission at a late step of cytokinesis $[8,9]$, and budding and release of some enveloped viruses from the plasma membrane of infected cells [10]. In Saccharomyces cerevisiae, the ESCRT-III protein complex comprises four core subunits: Vps20, Vps24, Vps2, and Snf7 (vacuolar sorting proteins 20, 24, 2, and sucrose non-fermenting protein 7), whereas, in Homo sapiens, up to 13 proteins form the ESCRT-III family called charged multivesicular body protein (CHMP1-8; IST1) (Additional file 1: Figure S1-A). The increased number of ESCRT-III subunits in Homo sapiens reflects the functional diversification of the complex in higher organisms [11].

(c) The Author(s). 2021 Open Access This article is licensed under a Creative Commons Attribution 4.0 International License, which permits use, sharing, adaptation, distribution and reproduction in any medium or format, as long as you give appropriate credit to the original author(s) and the source, provide a link to the Creative Commons licence, and indicate if changes were made. The images or other third party material in this article are included in the article's Creative Commons licence, unless indicated otherwise in a credit line to the material. If material is not included in the article's Creative Commons licence and your intended use is not permitted by statutory regulation or exceeds the permitted use, you will need to obtain permission directly from the copyright holder. To view a copy of this licence, visit http://creativecommons.org/licenses/by/4.0/ The Creative Commons Public Domain Dedication waiver (http://creativecommons.org/publicdomain/zero/1.0/) applies to the data made available in this article, unless otherwise stated in a credit line to the data. 
In yeast, the sequence of recruitment of ESCRT-III proteins during MVB formation is Vps20-Snf7-Vps24Vps2, forming a core complex [12]. Their human homologs are respectively CHMP6-CHMP4 (A, B, C)-CHMP3CHMP2 (A, B). Both CHMP2A and CHMP2B present a high sequence homology with the yeast protein $\mathrm{Vps} 2$ and have therefore been considered isoforms. Indeed, CHMP2B appears to be a relatively recent acquisition in the evolution of the ESCRT-III complex resulting from a Vps2 gene duplication event [11] (Additional file 1: Figure S1-B). Together, CHMP2A and CHMP2B act in most ESCRTcatalyzed membrane remodeling processes, except in MVB formation [5], where CHMP2A but not CHMP2B is required, and neuronal pruning which requires $\mathrm{CHMP2B}$ but not CHMP2A. Yet so far, the dual roles of CHMP2A and $\mathrm{CHMP} 2 \mathrm{~B}$ in the interaction and remodeling of membranes remain unclear [6, 13-17] (Additional file 1: Figure S1-C).

ESCRT-III proteins cycle between an inactive cytosolic state [18-20] and an activated state [21-23] leading to filamentous polymers forming spirals or helical tubular structures in vitro and in vivo [19, 24-42].

Purified recombinant CHMP2A can coil up into flat snail-like structures [43] or form helical tubular polymers with CHMP3 in the absence of membrane [25]. On the other hand, overexpressed CHMP2B in cells [32] leads to the formation of tubular helical structures, but in vitro assembly of recombinant CHMP2B has never been visualized, neither alone nor together with CHMP3. SiRNA knockdown of individual ESCRT-III proteins demonstrated a minimal requirement of one CHMP4 and one CHMP2 member for HIV-1 release [14]. Furthermore, CHMP3 acts synergistically with CHMP2A but not CHMP2B [44], indicating a distinct role for CHMP2B independently of CHMP3. In contrast, both CHMP2A and CHMP2B are important for cytokinesis [45]. So far, CHMP2A and CHMP2B have been considered as functional homologs, but practically no study has questioned yet whether CHMP2A and CHMP2B behave similarly upon binding to membranes to validate this hypothesis.

Biophysical in vitro membrane models, albeit their limitations, have provided important new insight into membrane remodeling processes in general $[46,47]$ as well as into ESCRT function, such as CHMP2B acting as a diffusion fusion barrier [48], the role of membrane curvature for ESCRT-III interaction [49, 50], ESCRT-III polymerization on supported lipid bilayers [30, 51], ESCRT-III polymerization on membranes [35], and ESCRT-catalyzed membrane fission [39, 52].

Here, we have investigated in vitro the functional homology of CHMP2A and CHMP2B in the ESCRT machinery, using biomimetic membrane systems with purified CHMP proteins. We have compared their membrane binding and their mechanical effects on membrane by confocal microscopy, flow cytometry (FACS), quartz crystal microbalance with dissipation monitoring (QCM-D), and high-speed atomic force microscopy (HS-AFM). We further investigated the role of charged lipids for membrane interaction as well as the role of CHMP3 on the polymerization of CHMP2A and CHMP2B, respectively. We confirm that CHMP3 works synergistically with CHMP2A for enhancing their mutual binding towards membranes, but reduces the binding of CHMP2B. We establish that CHMP2B binding is enhanced in the presence of PI(4,5)P2 lipids forming a protein network on the membrane surface, whereas CHMP2A+CHMP3 interact homogenously with membranes via electrostatic interactions without phosphoinositide binding specificity. Moreover, we study the mechanical properties of membranes coated with these different ESCRT assemblies. We show by micropipette aspiration, osmotic shock, and HS-AFM deformation that CHMP2A and CHMP2B have different mechanical effects on the membrane. While CHMP2B highly rigidifies membranes, CHMP2A+CHMP3 have almost no effect on it. Together, our study demonstrates that CHMP2A and CHMP2B cannot be considered as functional homologs. Thus, the observed different mechanical properties are likely important for understanding the mechanics of membrane remodeling and membrane scission.

\section{Results}

\section{CHMP2B and CHMP2A display different membrane}

\section{binding characteristics}

Phosphoinositides constitute a minority of the phospholipid family with a concentration lower than $1 \%$ in cell membranes. Nevertheless, PIP lipids play an essential role for signaling in cells. PI(3)P is the main phosphatidyl inositide present in the endosomal compartments of the MVB pathway where the ESCRTs were first identified, and this lipid has been used in purified systems to reconstitute MVB formation using yeast proteins [53]. However, ESCRT-III-mediated processes also occur on membranes enriched in $\mathrm{PI}(4,5) \mathrm{P} 2$, notably at the plasma membrane, including for instance HIV-1 egress, plasma membrane repair, and cytokinesis events, or at the nuclear envelope $[54,55]$. We have thus first compared the interactions of CHMP2A and CHMP2B with membranes containing different phosphatidyl-inositides. To improve protein solubilization, CHMP2A was fused to MBP. In order to exclude effects of the MBP fusion, CHMP2B function was analyzed in parallel to MBP-CHMP2B.

A previous in vitro study [48] has shown that the interaction of CHMP2B with membrane is significantly enhanced in the presence of $\mathrm{PI}(4,5) \mathrm{P} 2$ lipids in comparison with DOPS or PI $(3,5) P 2-$ membranes. Thus, we compared the preferential binding of CHMP2A versus 
CHMP2B on GUVs containing 10\% PI(4,5)P2 using confocal imaging.

$10 \%$ PI $(4,5)$ P2-GUV (see composition 1 in the "Methods" section) were incubated for $30 \mathrm{~min}$ with CHMP2A or CHMP2B proteins at a concentration of $500 \mathrm{nM}$ in the protein binding buffer (BP buffer), which has been optimized to ensure the highest protein density on the GUV membrane (Additional file 2: Figure S2-A). It has been shown that the displacement or truncation of the C-terminal region of CHMP proteins facilitates activation of ESCRT-III proteins polymerization on membranes [19, 21, 22, 48]. Thereupon, C-terminal truncated of MBP-CHMP2A- $\Delta \mathrm{C}$ and CHMP2B- $\Delta \mathrm{C}$ were used in the following experiments.

While CHMP2B- $\Delta$ C shows a homogenous binding to the GUV (Fig. 1a, first panel), the interaction of MBP-CHMP2A- $\triangle \mathrm{C}$ is rather weak under the same conditions (Fig. 1a, third panel). Although MBP cleavage increases the interaction, it also induces aggregation of CHMP2A- $\Delta \mathrm{C}$ in solution and on the membrane, resulting in the formation of aggregates containing lipids and proteins (Additional file 2: Figure S2-B). However, to test the effect of the MBP tag, we compared the properties of MBP-CHMP2B- $\Delta \mathrm{C}$ to that of CHMP2B- $\triangle \mathrm{C}$ and showed they have the same membrane binding properties and mechanical effect on the membrane (Additional file 2: Figure S2-C and $D$ ), indicating that the MBP fusion does not per se affect membrane binding.

Previous experiments have shown that in solution, combinations of MBP-CHMP2A- $\Delta \mathrm{C}$ and CHMP3- $\Delta \mathrm{C}$ as well as MBP-CHMP2A- $\triangle \mathrm{C}$ and CHMP3-FL co-polymerize to form tubular helical structures more efficiently than combinations of CHMP2A-FL and CHMP3-FL or CHMP2A-FL and CHMP3- $\triangle \mathrm{C}$ [25]. We have thus tested the effect of CHMP3-FL on the polymerization of MBP-CHMP2A- $\Delta$ C. In the following experiments, MBP-CHMP2A- $\Delta C$, CHMP2B- $\triangle \mathrm{C}$, and CHMP3-FL will be referred to as CHMP2A, CHMP2B, and CHMP3, respectively. After incubation of $10 \% \mathrm{PI}(4,5) \mathrm{P} 2-\mathrm{GUV}$ s with CHMP2A (or CHMP2B) + CHMP3 (500 nM and $2 \mu$ M respectively in BP buffer), we found that CHMP2A strongly binds to GUVs in the presence of CHMP3 (Fig. 1a, fourth panel). The quantification of the fluorescence intensity (see details in the "Methods" section) of CHMP2A on GUVs by confocal microscopy shows that the binding of CHMP2A to the membrane is increased by a factor of at least 2.5 in the presence of CHMP3 (Fig. 1b). Unexpectedly, when CHMP3 is incubated with CHMP2B, the binding of CHMP2B is no longer homogenous and appears as patches on the GUV colocalizing with CHMP3 (Fig. 1a, second panel). The relative amount of CHMP2B on the membrane is decreased by a factor of 2 as compared to the relative
CHMP2B amount measured in the absence of CHMP3 (Fig. 1b).

To quantify the amount of protein bound to the GUV membrane with higher statistics, we have used flow cytometry (FACS) [56] and 2\% PI(4,5)P2-GUVs incubated with a combination of CHMP2A or CHMP2B with and without CHMP3, respectively, at $500 \mathrm{nM}$ for both CHMP2A and CHMP2B proteins and $2 \mu \mathrm{M}$ for CHMP3 for $30 \mathrm{~min}$. The fluorescence intensity of the membrane and of the proteins is proportional to the amount of fluorophores in the membrane and proteins bound to it or present in the detection zone, respectively. From the plot of the protein intensity versus lipid signal for all recorded events, we could determine the signals corresponding to CHMP proteins bound to GUVs and plot the corresponding histogram of these intensities for the different conditions. The median value of this histogram is related to the average density of proteins bound to GUVs. When CHMP2A or CHMP3 are incubated alone with the $2 \%$ $\mathrm{PI}(4,5) \mathrm{P} 2-G U V$ suspension, an extremely weak signal is detected by FACS. However, as previously observed by confocal microscopy, binding increases significantly by almost 100x, when both proteins are incubated together, in comparison to CHMP2A alone (Fig. 1c). On the contrary, the presence of CHMP3 decreases the binding efficiency of CHMP2B-by approximately $150 \%$ (Fig. 1c). Previous experiments with surface plasma resonance (SPR) have studied the interactions of MBP-CHMP2A- $\Delta \mathrm{C}$ with CHMP3 and CHMP2B with CHMP3 in solution [44]. CHMP2A and CHMP3 interacted with a $K_{\mathrm{D}}$ of $3.2 \mu \mathrm{M}$ and CHMP2B-CHMP3 interacted with a $K_{\mathrm{D}}$ of $1.4 \mu \mathrm{M}$. In the present work, the protein concentrations are lower than these $K_{\mathrm{D}}$ values. As a consequence, CHMP2A and CHMP2B have a stronger interaction with the membrane than their mutual interaction. We conclude that CHMP2A and CHMP3 synergize in binding to membranes, while CHMP3 might act as a negative regulator for CHMP2B membrane binding in vitro.

All the previous experiments were performed with GUVs containing PI(4,5)P2 lipids. In vivo, membranes are enriched with different PIP species depending on their localization. We thus wondered if the behavior of CHMP2A and CHMP2B in the presence or absence of CHMP3 would be affected by the incorporation of other phosphoinositides in the membrane. In the following, we tested the effect of PIP specificities and the effect of the charge. We thus performed experiments at a constant PIP fraction/concentration or at a constant charge ratio.

GUVs were produced with $2 \%$ of $\mathrm{PI}(3) \mathrm{P}, \mathrm{PI}(3,5) \mathrm{P} 2$, PI(4)P, or PI $(4,5)$ P2 (see composition 2 in the "Methods" section), which are enriched at the early endosomes, late endosomes, endoplasmic reticulum/Golgi, and plasma membrane, respectively [54]. They were then incubated with $\mathrm{CHMP2A}+\mathrm{CHMP3}$ or CHMP2B alone or in 


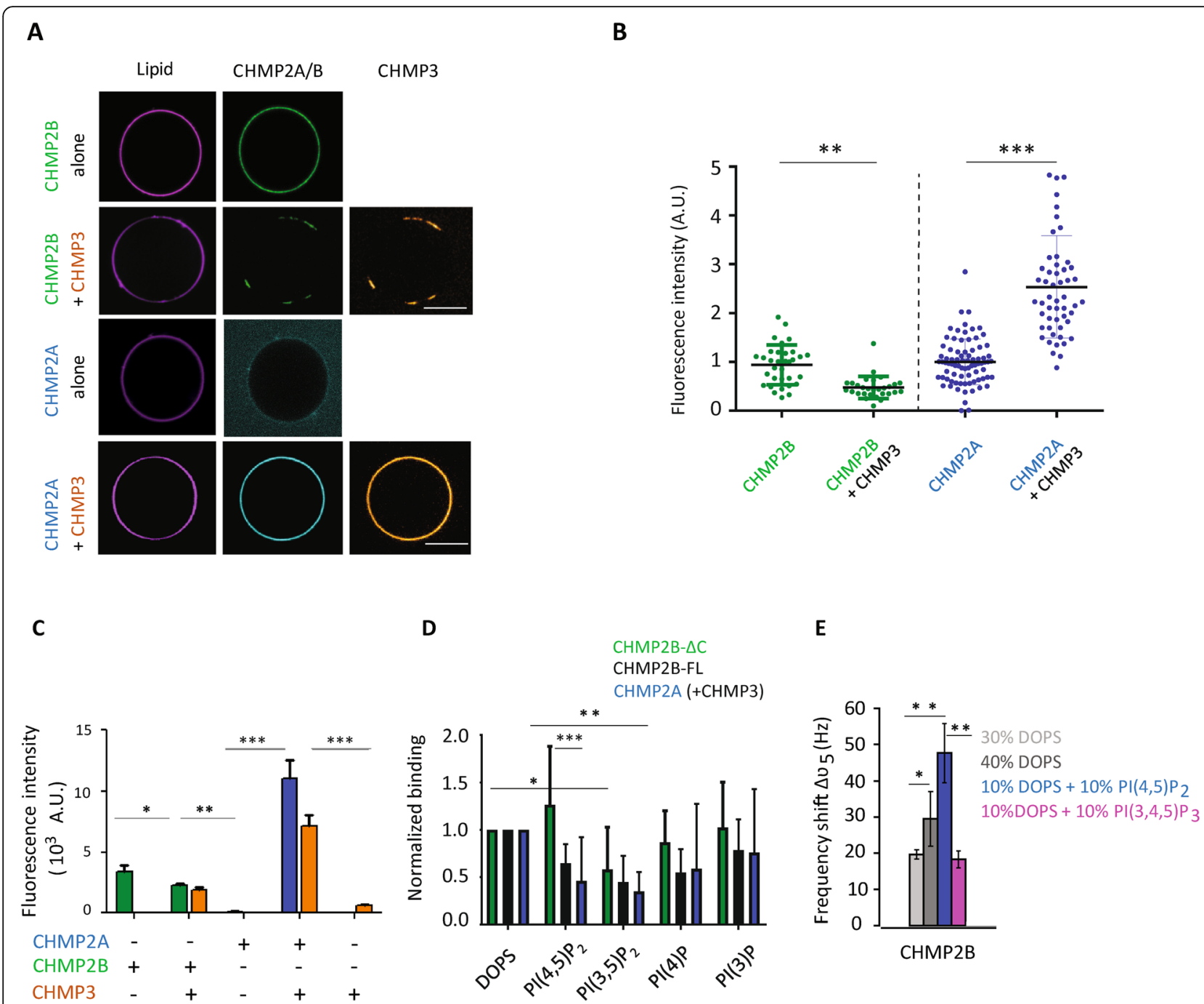

Fig. 1 Interaction of CHMP2A versus CHMP2B with charged model membranes. a Confocal images of 10\% PI (4,5)P2-GUVs incubated with 500 nM CHMP2B- $\triangle$ C (first line) (called CHMP2B) and MBP-CHMP2A- $\triangle$ C (third line) (called CHMP2A) alone or in combination with $2 \mu M C H M P 3$ (second and fourth lines, respectively). A single confocal plane is shown. Scale bar, $10 \mu \mathrm{m}$. Note that in the case of MBP-CHMP2A- $\Delta C$ (third line), the laser intensity in the protein channel has been increased (as visible by the higher background intensity) to detect protein binding on the GUV membrane. $\mathbf{b}$ Effect of CHMP3 on MBP-CHMP2A- $\Delta$ C and CHMP2B- $\Delta$ C binding to 10\% PI (4,5)P2-GUVs (same conditions as in a). The fluorescence intensity was measured from the analysis of spinning disk microscopy images using the Cell Profiler software. The fluorescence intensity of MBP-CHMP2A- $\triangle C+C H M P 3$ and CHMP2B- $\triangle C+C H M P 3-$ covered vesicles was normalized to the intensity of MBP-CHMP2A- $\triangle C$ and CHMP2B- $\Delta C$-covered vesicles, respectively. ${ }^{* *} p$-value $<0.01 ;{ }^{* * *} p$-value $<0.001$ (Student's $t$-test). $N=48$. c Quantification by FACS of the fluorescence intensities of MBP-CHMP2A- $\Delta \mathrm{C} \pm$ CHMP3, CHMP2B- $\Delta \mathrm{C} \pm$ CHMP3 and CHMP3 co-polymers bound to 10\% PI(4,5)P2-containing GUVs. The concentrations of CHMP2A/B and CHMP3 proteins are, respectively, $500 \mathrm{nM}$ and $2 \mu \mathrm{M}$. ${ }^{*} p$-value $<0.05 ;{ }^{* *} p$-value $<0.01$; ${ }^{* * *} p$-value $<0.001$ (Student's t-test). $N=4$ (number of FACS experiment with about $10^{4}$ counted events per experiment, per condition). d Quantification of CHMP2B$F L, C H M P 2 B-\triangle C$, and MBP-CHMP2A- $\triangle C$ (CHMP2A) + CHMP3 binding to GUVs containing DOPS and different PIPs by flow cytometry (FACS). Equimolar amount of DOPS and different PIPs ( $2 \% \mathrm{~mol} / \mathrm{mol}$ of total lipids) have been used. Note that data on CHMP2B- $\Delta C$ binding to DOPS, $\mathrm{PI}(4,5) \mathrm{P} 2$, and $\mathrm{PI}(3,4) \mathrm{P} 2$ were already published [48]. Binding efficiencies were normalized to the fluorescence intensity of DOPS-containing vesicles (see Figure S2.D). These values were then normalized to the total amount of charge for each lipid composition. ${ }^{*} p$-value $<0.05$; ${ }^{* *} p$-value $<0.01$; ${ }^{* * *} p$-value $<0.001$ (Student's $t$-test). $N=6$ (number of FACS experiment with about $10^{4}$ counted events per experiment, per condition). e Resonance frequency shift $\vartheta_{5}$ in the QCM-D experiments when CHMP2B- $\Delta$ C is bound to the different types of supported lipid bilayers (light gray 30\% DOPS, 70\% DOPC; gray 40\% DOPS, 60\% DOPC; light blue 10\% DOPS, 10\% PI(4,5)P2, 80\% DOPC, magenta 10\% DOPS, 10\% PI(3,4,5)P3, 80\% DOPC). ${ }^{*} p$-value $<0.05 ;{ }^{* *} p$-value $<0.01 ;{ }^{* * *} p$-value $<0.001$ (Student's $t$-test). $N=5$

combination with CHMP3 for 30 min to optimize the protein coverage on the membrane. The amount of protein bound to the GUV membrane was analyzed by
FACS. The median values of the histograms of binding efficiency for the different PIP species (Additional file 3: Figure S3-A) were normalized by the mean value of the 
distribution of proteins bound to DOPS vesicles (control GUV without PIPs) (Additional file 3: Figure S3-B). The binding of CHMP2A+CHMP3 is almost identical, within the error bars, for all the conditions tested when GUVs are doped with a nominal constant fraction of phosphoinositide.

To test the charge effect, the binding efficiencies have been normalized by the charge of each PIP species, considering that DOPS has a net charge of $-1, \mathrm{PI}(4,5) \mathrm{P} 2$ (or PI(3, 5)P2) of -3 at $\mathrm{pH} 7.5$, and $\mathrm{PI}(4) \mathrm{P}$ (or $\mathrm{PI}(3) \mathrm{P}$ ) of -2 [57] (Fig. 1d). The binding of CHMP2A+CHMP3 is almost identical for all PIP species, except for PI $(3,5) \mathrm{P} 2$, which shows a decrease by a factor of $2.8(p$-value $=0.008)$ in comparison to DOPS GUVs (Fig. 1d). After charge renormalization, the binding of CHMP2B is identical for GUVs containing DOPS and all types of PIP species tested, except for PI(3,5)P2 (Fig. 1d). We did not measure a significantly higher binding efficiency of CHMP2B for PI $(4,5) \mathrm{P} 2$ membranes than for pure DOPS membranes (Fig. 1d, $p$ value $=0.04$ ); nevertheless, for this specific composition, binding is much stronger for CHMP2B than CHMP2A+ CHMP3 (Fig. 1d). Indeed, the binding of CHMP2B to PI(4, 5) $\mathrm{P} 2$ containing membranes is 2.8 times higher than the binding of CHMP2A+CHMP3 ( $p$-value $=0.03$ ). The binding of CHMP2B is almost doubled on PI(4,5)P2 membranes compared to $\mathrm{PI}(3,5) \mathrm{P} 2$ ( $p$-value between $\mathrm{PI}(4,5) \mathrm{P} 2$ and $\mathrm{PI}(3,5) \mathrm{P} 2 \mathrm{GUVs}=0.03)$. In contrast, we did not observe such a preference for CHMP2B-FL (Fig. 1d).

In summary, employing GUVs with a nominal constant concentration of PIP species, $\mathrm{CHMP} 2 \mathrm{~A}+\mathrm{CHMP} 3$ do not seem to exert a specificity for PIP, whereas CHMP2B shows a strong increase in its binding capacity in the presence of PI(4,5)P2 before charge normalization. In addition, after charge normalization, the binding efficiency of CHMP2B and CHMP2A+CHMP3 is lower for $\mathrm{PI}(3,5) \mathrm{P} 2$.

One of the main difficulties when working with $\mathrm{PI}(4$, 5)P2 GUV is that this lipid can exchange with the surrounding bulk $[54,55]$. We thus checked with a complementary technique whether variations in PIP concentrations might have affected ESCRT-III interaction with GUV membranes. For this purpose, we used the quartz crystal microbalance with dissipation monitoring (QCM-D) to measure CHMP2B binding to supported lipid bilayers (SLBs) with a constant net charge fraction (see the "Methods" section). Indeed, the fraction of charged lipids is well preserved during SLB preparation from fusion of small unilamellar vesicles (SUVs) onto solid substrates [58]. After SLB formation with a defined DOPS or PIP composition (see lipid compositions in the "Methods" section), CHMP2B proteins were added in the chamber resulting in a shift of the resonance frequency $\Delta 9_{5}$ of the quartz sensor, directly related to the amount of protein bound to the surface
(Additional file 3: Figure S3-C). The amount of proteins adsorbed to the bilayer increased by $50 \%$ when the amount of DOPS was increased from 30 to $40 \%$ (Fig. $1 \mathrm{e})$. Indeed, increasing the number of negatively charged lipids in the membrane increases the amount of protein adsorbed on it. This implies that electrostatic interactions play a key role in mediating the interaction between the proteins and the membrane in agreement with the exposure of basic surfaces in ESCRT-III polymers [35, 37]. Furthermore, in order to discriminate between the specific affinity for PI(4,5)P2 lipids and electrostatic interactions, we prepared SLBs with a constant total net charge with either $40 \%$ DOPS or $10 \%$ DOPS $+10 \% \operatorname{PI}(4,5) \mathrm{P} 2$, the total net charge of these SLBs being equivalent. We observe that CHMP2B density is approximately $60 \%$ higher when $\mathrm{PI}(4,5) \mathrm{P} 2$ lipids are present in comparison with SLBs made of DOPS only (Fig. 1e). Compared to experiments on GUVs (Fig. 1d), this higher enhancement is probably due to an effective higher PI(4, 5)P2 fraction in the SLBs as compared to the GUVs. Moreover, when PI(4,5)P2 lipids are replaced by the same fraction of $\mathrm{PI}(3-5) \mathrm{P} 3$, the amount of proteins bound to the SLB decreases significantly and becomes almost equal to the amount of proteins bound to SLB with 30\% DOPS only, although PI(3-5)P3 lipids have a higher negative net charge $(-4)$ as compared to PI(4,5)P2 lipids $(-3)[57,59]$. Altogether, these experiments show that CHMP2B preferentially interacts with PI(4,5)P2 lipids.

Globally, our results show that while CHMP2B is capable of binding to membrane alone, membrane binding of CHMP2A is significantly enhanced by CHMP3 (Fig. 1b, c). Additionally, CHMP3 has a modulating effect on CHMP2B and reduces its membrane association (Fig. 1b, c). Moreover, we found that the binding of CHMP2A+CHMP3 does not depend on the PIP species present in the membrane composition, in contrast to the enhanced binding of CHMP2B in the presence of PI(4,5)P2 lipids. This nonspecificity of CHMP2A (+CHMP3) proteins to any of the PIP species including PI(4,5)P2 is in agreement with their presence in most cellular processes involving the ESCRTIII complex [1], contrary to CHMP2B which is only required for processes occurring at the plasma and nuclear membranes that are enriched in PI(4,5)P2 lipids $[3,60,61]$.

\section{CHMP2A and CHMP2B exhibit different supramolecular assemblies on membranes}

Previous studies have shown that cellular overexpression of CHMP2B leads to helical scaffolds deforming the plasma membrane into long rigid tubes protruding out of the cell [32]. Similarly, CHMP2A+CHMP3 coassemble in bulk into helical tubes in vitro $[25,44]$ or helical filaments on membrane tubes [41, 62]. Because the characterization of the effect of ESCRT-III on deformable model membranes is crucial to understand 
their mechanical properties, we further studied the supramolecular assemblies of CHMP2B versus CHMP2A+CHMP3 on $10 \% \mathrm{PI}(4,5) \mathrm{P} 2-\mathrm{GUV}$ s by spinning disk confocal microscopy.

Above $500 \mathrm{nM}$ protein bulk concentration, CHMP2B proteins fully cover the surface of GUVs with no observable distinctive structure, i.e., no inward or outward tubulation (Fig. 2a, first panel). At optical resolution, CHMP2B proteins appear homogeneously distributed on the surface of the vesicles, besides some protein-lipid patches. At bulk concentration lower than $500 \mathrm{nM}$, CHMP2B proteins form a peculiar reticular-like network wrapping around the whole vesicle (Fig. 2a, second panel). Notably, the same network is observed when MBP-CHMP2B- $\Delta$ C is used (Additional file 2: Figure S2$\mathrm{C}$ ), indicating that the MBP fusion does not affect its function. The networks were observed after 15-min GUV incubation in the protein solution suggesting that, at high bulk concentration, a reticulum-like network forms transiently, becoming denser with time and leading to an apparent continuous coverage at optical resolution. This CHMP2B network co-localizes with PI(4, 5)P2 lipids (Fig. 2b), indicating that CHMP2B recruits negatively charged $\mathrm{PI}(4,5) \mathrm{P} 2$ lipids, further confirming the specific interaction between CHMP2B and PI $(4,5) \mathrm{P} 2$ lipids.

In contrast, the assembly of CHMP2A+CHMP3 appears to be devoid of any visible network, independent of the incubation time and protein concentration (up to $2 \mu \mathrm{M}$ of CHMP2A) (Fig. 2c and Additional file 3: Figure S3-D). In some vesicles (approx. 10\%), we observed CHMP2A (+ non-labeled CHMP3)-containing short, outward protrusions (Fig. 2c, and zoom-in). These protrusions were, however, rarely visible on most of the vesicles. We conclude that CHMP2B and CHMP2ACHMP3 do not tubulate GUV membranes in this concentration range.

We next investigated whether these proteins perturb the mechanical properties of the membranes.

\section{CHMP2A and CHMP2B have different mechanical effects on model membranes}

To study the mechanical effect of CHMP2B and $\mathrm{CHMP} 2 \mathrm{~A}+\mathrm{CHMP} 3$ on membranes, we first used the micropipette aspiration technique developed by E. Evans [63], to measure the elasticity of $10 \% \mathrm{PI}(4,5) \mathrm{P} 2-\mathrm{GUV}$ (lipid composition 1) coated with CHMP2A or CHMP2B in the presence or absence of CHMP3.

In the absence of CHMP proteins, micropipette aspiration of PI(4,5)P2-GUVs easily induced the formation of a characteristic tongue inside the micropipette (Fig. 3a, first panel). In contrast, $\mathrm{PI}(4,5) \mathrm{P} 2-\mathrm{GUV}$ s incubated with a CHMP2B concentration leading to full coverage could not be aspirated and deformed even at high tensions
(Fig. 3a, second panel) (up to $10^{-3} \mathrm{~N} \mathrm{~m}^{-1}$ ). However, during aspiration at high tensions, in approximately $20 \%$ of the aspirated GUVs (Fig. 3b), an occasional rupture of CHMP2B protein coat could be observed, allowing the formation of a short tongue devoid of proteins inside the micropipette (Fig. 3a, third panel). This observation indicates that the CHMP2B polymer itself cannot be aspirated nor deformed and behaves as a solid shell. Surprisingly, the subsequent CHMP3 incubation with GUVs with pre-formed CHMP2B polymers on their surface resulted in the softening of the CHMP2B shell, which allowed aspiration of the GUV (Fig. 3a, fourth panel). The quantification of the percentage of aspirated vesicles at a tension of approximately $10^{-3} \mathrm{~N} \mathrm{~m}^{-1}$ clearly indicates that while less than $20 \%$ of the CHMP2Bcoated GUVs could be aspirated in the absence of CHMP3, generally upon shell rupture, almost $100 \%$ of the CHMP2B-coated GUVs could be aspirated when CHMP3 proteins were added (Fig. $3 b$ ). This thus suggests that CHMP2B polymers form a rigid shell around the vesicle that cannot be deformed by aspiration even at tensions as high as a few $10^{-3} \mathrm{~N} \mathrm{~m}^{-1}$ unless CHMP3 is present. The presence of CHMP3 softens this rigid shell allowing its deformation by the micropipette.

In contrast, $\mathrm{PI}(4,5) \mathrm{P} 2$-GUVs co-incubated with CHMP2A+CHMP3 could be easily deformed during aspiration with an increase of the tongue length inside the micropipette as a response to the aspiration increase (Fig. 3a, fifth panel). Figure 3c shows the variation of the membrane tension as a function of the fractional excess area, $\Delta \alpha$, for two representative experiments. The stretching modulus, $\chi$, is calculated from the slope of all the curves for both conditions (for bare lipids and membrane covered with CHMP2A+CHMP3; see the "Methods" section). The stretching modulus (Fig. 3d) is slightly decreased by the presence of CHMP2A+CHMP3 on the membrane. It is found to be equal to $X=11 \pm 6$ $\mathrm{mN} \mathrm{m}^{-1}(N=30$ GUVs$)$ for CHMP2A+CHMP3-covered GUVs and $X=26 \pm 19 \mathrm{mN} \mathrm{m}^{-1}(N=20$ GUVs $)$ for protein-free GUVs. This slight decrease means that CHMP2A+CHMP3 renders the GUV membrane slightly more stretchable. Note that the value of the stretching modulus for the bare lipid membrane is lower than values reported for dioleoyl, DO, chains, in the presence of cholesterol [64], probably because of the absence of a pre-stretching step in our experiments, as usually performed to suppress any pre-existing uncontrolled excess area [64]. Here, pre-incubation of the GUVs with proteins prevented any pre-stretching of the GUVs in order to limit the contact between pipette and protein-coated GUV and thus adhesion. Nevertheless, our aim was not to measure the absolute stretching modulus of the membranes coated by ESCRTs but to perform measurements relatively to bare lipid membranes in the same 
A

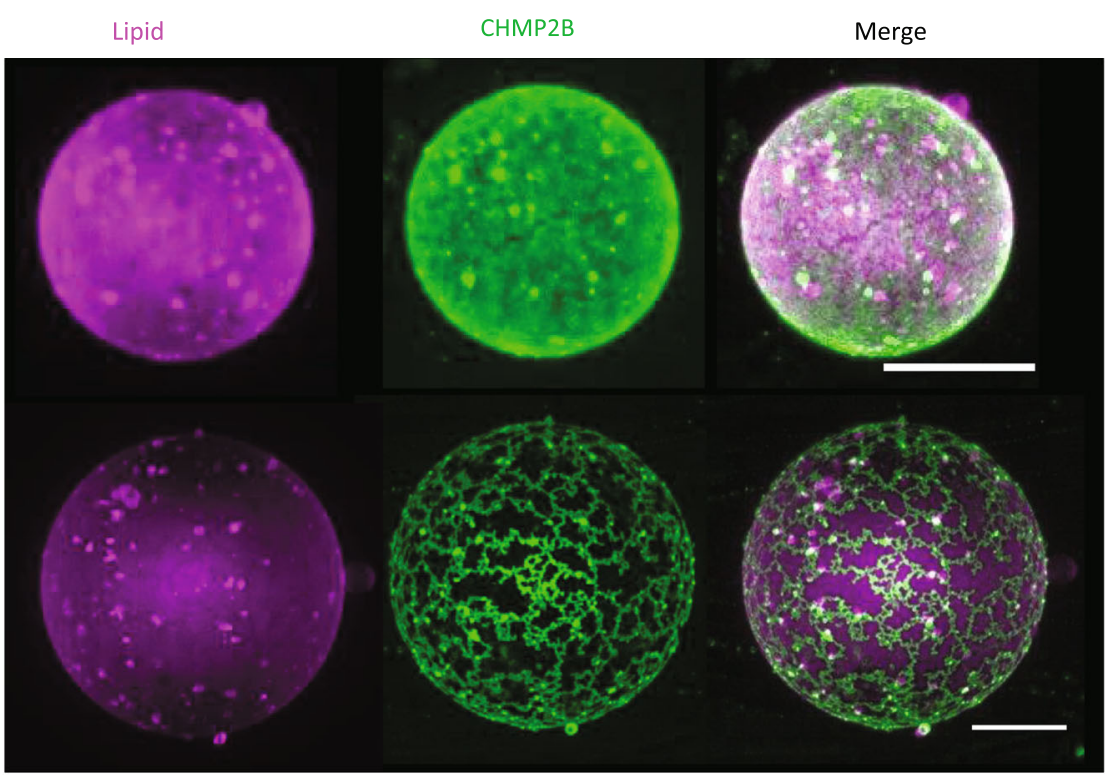

B Fluo- $P_{2}(4,5) P$

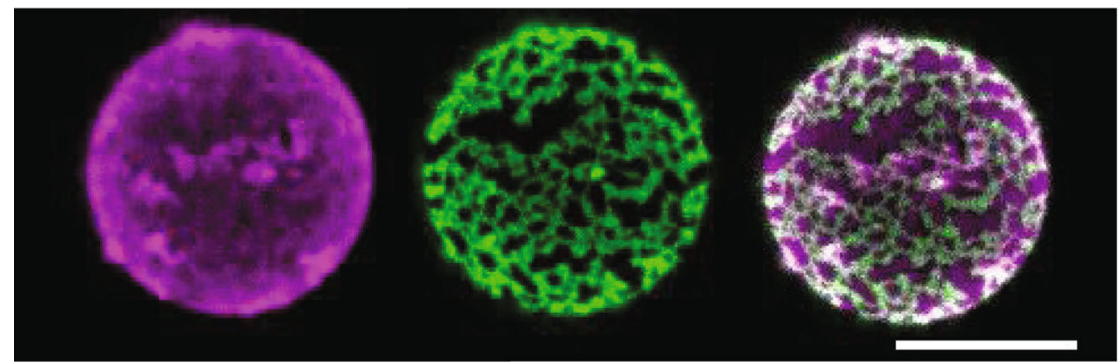

C

Lipid

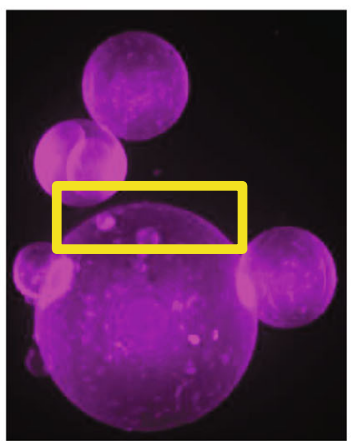

CHMP2A

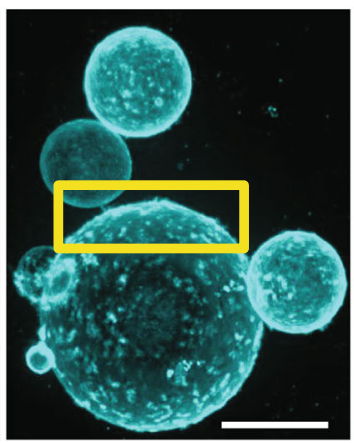

Zoom-in

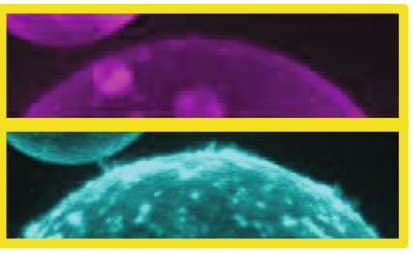

Fig. 2 Supramolecular assemblies of CHMP2A+CHMP3 versus CHMP2B on GUVs. a Spinning disk images of supramolecular assemblies of CHMP2B- $\triangle$ C (called CHMP2B) in BP buffer on 10\% PI(4,5)P2-GUVs. After 15-min incubation of the GUVs with the protein solution, CHMP2B- $\Delta$ C at high bulk protein concentration (1 $\mu \mathrm{M}$ ) (first panel) fully covers the vesicle surface, whereas at lower protein concentration (500 nM), CHMP2B- $\Delta C$ assembles into a reticular-like network on the GUV (second panel). A z-projection of the whole GUV is shown. Scale bar, $10 \mu \mathrm{m}$. b Co-localization of Fluo-PI(4,5)P2 and CHMP2B- $\Delta$ C on GUVs. A z-projection of the upper part of the GUV is shown. Scale bar, $10 \mu m$. c Spinning disk images of supramolecular assemblies of MBP-CHMP2A- $\triangle \mathrm{C}(500 \mathrm{nM})+\mathrm{CHMP3}(2 \mu \mathrm{M})$ in BP buffer on 10\% PI(4,5)P2-GUVs. MBP-CHMP2A- $\triangle \mathrm{C}$ (called CHMP2A) fluorescent signal is displayed. After 60-min incubation, the co-polymer covers the vesicle surface in a homogeneous manner with the presence of some protrusions at the surface of the GUV (zoom-in). A z-projection is shown including a zoom-in in the right panel, showing short protrusions at the surface of the GUV. Scale bar, $10 \mu \mathrm{m}$ 


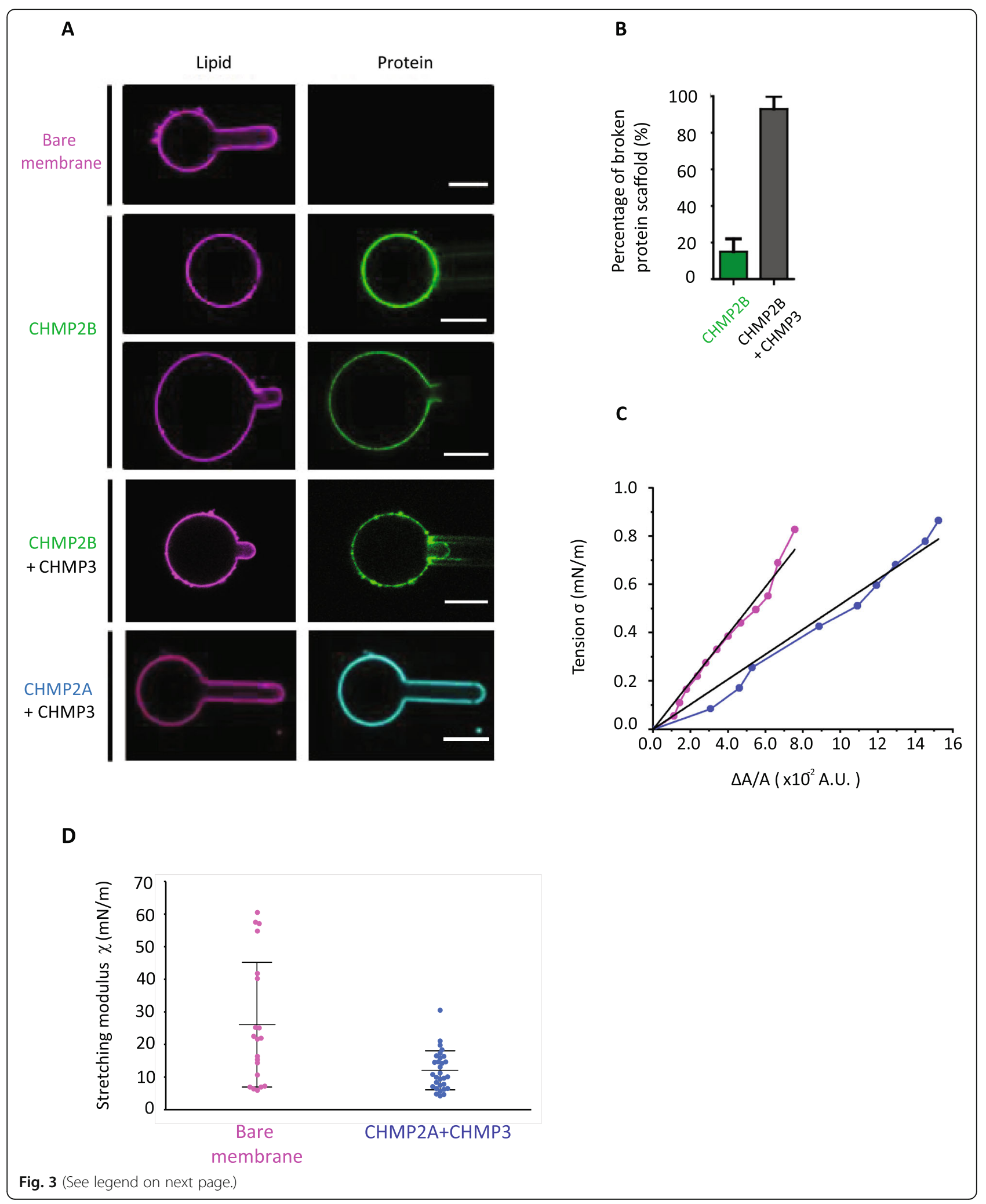


(See figure on previous page.)

Fig. 3 Mechanical properties of GUVs in the presence of CHMP2B versus CHMP2A+CHMP3 measured by micropipette aspiration. a Representative confocal single-plane images of micropipette aspiration performed at $\sigma \approx 5.10^{-3} \mathrm{~N} \mathrm{~m}^{-1}$ on a bare GUV containing $10 \% \mathrm{PI}(4,5) \mathrm{P} 2$ - (first panel), and on GUVs coated with CHMP2B- $\Delta$ C alone (second panel), CHMP2B- $\Delta C+C H M P 3$ (fourth panel), and MBP-CHMP2A- $\Delta C+C H M P 3$ (fifth panel) ( $C H M P 2 B$ corresponds to CHMP2B- $\triangle$ C and CHMP2A to MBP-CHMP2A- $\triangle$ C). The occasional rupture of CHMP2B polymer at high tension $\left(\sigma \approx 10^{-3}\right.$ $\mathrm{N} \mathrm{m}^{-1}$ ) is shown (third panel). Scale bar, $10 \mu \mathrm{m} . \mathrm{N}=30$ per condition. b Percentage of aspirated GUVs at $\sigma \approx 10^{-3} \mathrm{~N} \mathrm{~m}^{-1}$ with formation of a tongue inside the micropipette. Comparison between CHMP2B- $\Delta$ C-only GUVs and GUVs coated with CHMP2B- $\Delta C+C H M P 3 . N=14$ per condition. c Characteristic curves of the variation of the excess area $\triangle \mathrm{A} / \mathrm{A}$ as a function of the applied tension for a bare GUV (magenta) and a GUV coated with MBP-CHMP2A- $\triangle C+C H M P 3$ proteins (blue). The linear fit of each curve is represented (black). $\mathbf{d}$ Box plot of the stretching modulus for bare GUV ( $N=20$ experiments; magenta) or in the presence of MBP-CHMP2A- $\triangle C+C H M P 3$ ( $N=30$ experiments; blue) interacting with the GUV membrane $(p$-value $=0.002)$

experimental conditions. Moreover, the stretching modulus of membrane covered with $\mathrm{CHMP} 2 \mathrm{~B}+\mathrm{CHMP} 3$ could not be measured as the tongue covered with these proteins systematically adhered to the pipette, thereby impeding any mechanical measurement. We conclude that $\mathrm{CHMP} 2 \mathrm{~B}$ strongly rigidifies membranes, whereas $\mathrm{CHMP} 2 \mathrm{~A}+\mathrm{CHMP} 3$ membrane interaction does not alter the elastic properties of membranes.

We next applied different mechanical constraints to CHMP2B-covered GUVs to test their resistance to mechanical deformation. Spherical GUVs change shape when they are deflated upon an hyperosmotic shock since the surface/volume ratio increases and even becomes unstable when the osmotic shock is too strong [65]. We thus studied the effect of an hyperosmotic shock on $10 \% \mathrm{PI}(4,5) \mathrm{P} 2-\mathrm{GUV}$ s fully covered with CHMP2B by increasing the osmolarity in the external medium by salt or sugar addition. We carefully checked that the buffer change did not induce dissociation of the ESCRT-III proteins from the membrane. An osmotic shock equal to $150 \%$ (osmolarity of the external medium $\geq 190 \mathrm{mOsm} \mathrm{L} \mathrm{L}^{-1}$ ) transforms spherical protein-free GUVs into elliptical vesicles (Fig. 4a) with an average eccentricity index equal to $0.72 \pm 0.11$ (Fig. $4 \mathrm{~b}$ ) (note that the eccentricity index is the ratio between foci distance and the major axis length of an ellipse. It ranges between 0 (for a circle) and 1 (for a linear segment)). At higher osmotic shock, GUVs were completely destabilized in the absence of proteins. On the other hand, CHMP2Bcovered GUVs better preserved their spherical shape for the same $150 \%$ osmotic shock (Fig. 4a) with an average eccentricity index equal to $0.35 \pm 0.03$ (Fig. 4b). This behavior is not modified by the presence of the MBP tag (Additional file 2: Figure S2-D). Moreover, in contrast with bare membranes, vesicles covered with $\mathrm{CHMP} 2 \mathrm{~B}$ proteins could even stand a $300 \%$ osmotic shock in a solution at $500 \mathrm{mOsm} \mathrm{L}^{-1}$, showing again that $\mathrm{CHMP2B}$ polymer assembly on the GUV surface preserves vesicles from deformation by forming a rigid shell.

We next aimed to determine the effect of the different CHMP proteins on the mechanical properties of membranes at the nanometer scale. To do this, we applied a high-speed-AFM imaging-based deformation approach using small unilamellar vesicles (SUVs) with a typical diameter between 50 and $100 \mathrm{~nm}$.

First, a difference in surface roughness is observed between the SUV covered or not with the proteins. Whereas bare SUVs show a smooth surface, CHMPcoated vesicles possess a rougher surface, indicating the presence of the protein on the outside of the vesicles (Fig. 4c, top panels).

Next, we increased the imaging force and observed that the vesicles are progressively more deformed. The deformation of the SUVs is measured by recording the height change (Fig. 4c and Additional file 4: Figure S4). To assure that the vesicles had undergone elastic deformation, even at the maximum applied imaging force, the imaging force was reduced again to the minimum value at the end of the experiments. Only vesicles that bounced back to more than $90 \%$ of the initial height were considered for the analysis, and typically, the vesicles did recover their shape and size (Additional file 5: Movie S1). In Additional file 6: Figure S5, we show the raw data points for bare SUVs (Additional file 6: Figure S5-A), the transformation from absolute height to relative height (Additional file 6: Figure S5-B and C), and the raw data points for the relative height versus force increment (Additional file 6: Figure S5-D and E). The slope of these curves is directly related to the flexibility of the SUV membrane coated with the protein (Fig. 4d). Indeed, for a given force, a stiff membrane is less deformed than a soft one and the slope is lower. Figure 4d shows a clear difference between the different vesicles: The presence of CHMP2B stiffens the membrane by a factor $2.9 \pm 0.3$. However, the addition of CHMP3 reduces the stiffening effect of CHMP2B and the corresponding membranes with CHMP2B and CHMP3 are only $1.7 \pm 0.2$ times stiffer than the bare ones. In contrast, the presence of CHMP2A+CHMP3 does not modify the membrane elasticity as there is no significant difference compared to the slope of bare vesicles.

We conclude that CHMP2B stiffens membranes while the presence of CHMP3 renders CHMP2B-bound membranes more deformable. This indicates that CHMP3 may counteract the effect of CHMP2B on the mechanical properties of model membranes. In contrast, the 


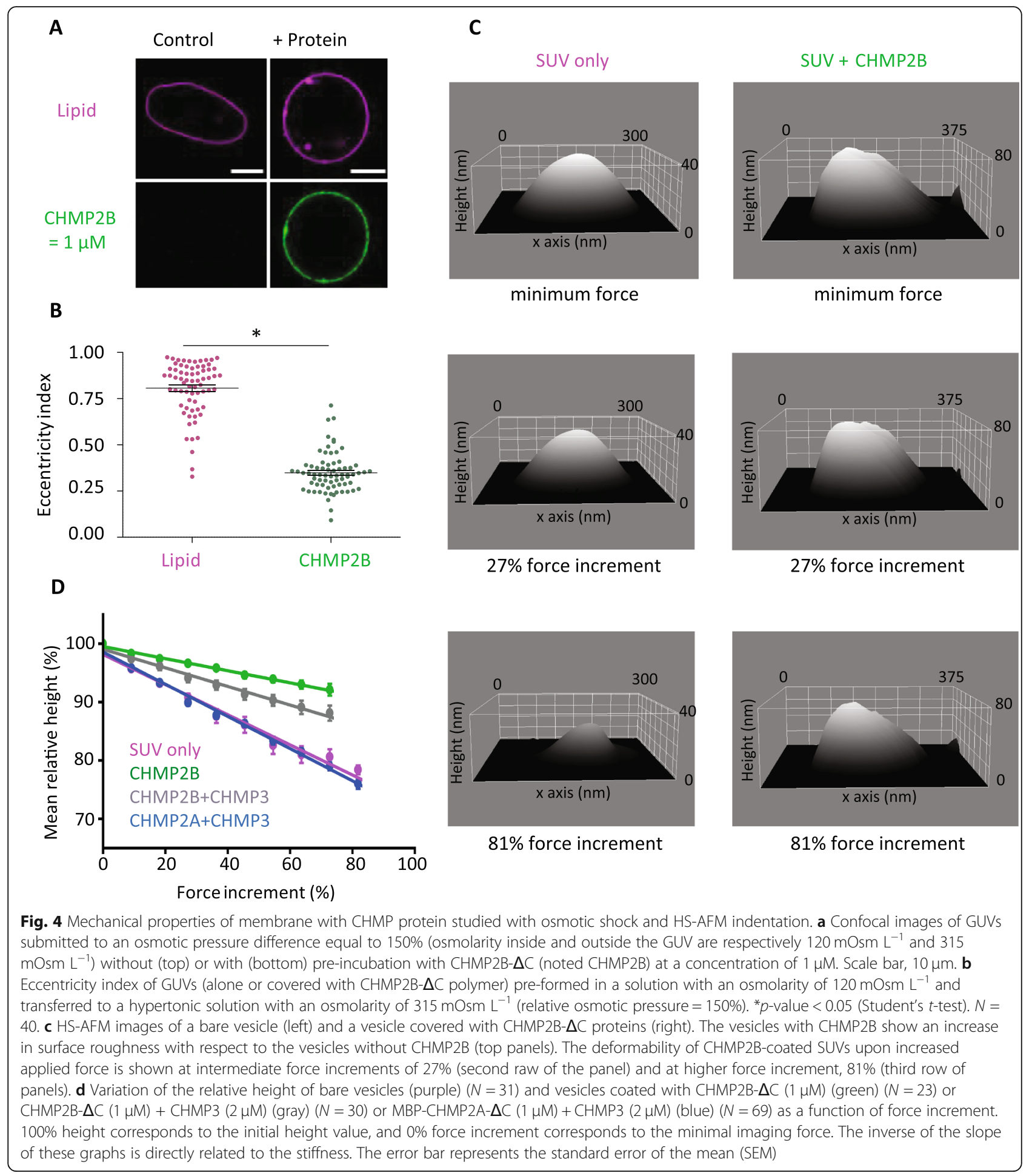

presence of CHMP2A+CHMP3 does not affect the mechanical properties of model membranes.

\section{Discussion}

The objective of our study was to compare the membrane binding properties of ESCRT-III proteins
CHMP2A and CHMP2B in vitro in order to determine their capacity to substitute each other during membrane remodeling processes.

First, we show that CHMP2A membrane binding is strongly enhanced in the presence of CHMP3, in agreement with previous in vivo and in vitro studies [23, 26, 
44, 66], whereas CHMP2B shows efficient membrane binding in the absence of CHMP3. This is in agreement with the synergy exerted by CHMP3 in the presence of CHMP2A and with the absence of synergy exerted by CHMP3 in the presence of CHMP2B on HIV-1 budding $[25,26,44,66]$. Our data further shows that the presence of CHMP3 confines CHMP2B to patches on the GUV membrane. Reduced membrane binding may be explained by a negative regulatory function of CHMP3 on CHMP2B, whereas CHMP3 interaction with CHMP2B [44] may have a similar function than $\mathrm{CC} 2 \mathrm{D} 1 \mathrm{~A} / \mathrm{B}$ or $\operatorname{lgd}$ in the negative regulation of CHMP4B/Snf7 polymerization [67-69]. Furthermore, the incubation of CHMP2B with CHMP3 in solution does not induce CHMP2B polymerization in solution, eliminating the possibility that premature CHMP2B polymerization is responsible for reduced $\mathrm{CHMP} 2 \mathrm{~B}$ membrane binding in the presence of CHMP3 (Additional file 7: Figure S6).

CHMP2A was N-terminally fused to MBP, which keeps CHMP2A monodisperse and monomeric in solution, while removal of MBP triggers spontaneous polymerization or aggregation. Since we observed the same membrane binding and mechanical properties of CMP2B- $\Delta$ C and MBP-CHMP2B- $\Delta$ C, our results suggest that the presence of MBP tag does not affect CHMP2A membrane binding either. In addition, many live-cellimaging experiments performed with $\mathrm{N}$-terminal fusions of ESCRT-III proteins show no major effect on the physiological function of ESCRT-III polymers containing fusion proteins $[8,70-73]$. Second, we confirm that CHMP2B displays a stronger binding for PI $(4,5) \mathrm{P} 2$ containing membranes as compared to other phosphoinositides and DOPS lipids [48]. In contrast, CHMP2A and CHMP3 require only negatively charged membranes for binding with no preference for specific lipid head groups. The binding affinity for $\mathrm{PI}(4,5) \mathrm{P} 2$ lipids is in agreement with the spontaneous localization of CHMP2B to the plasma membrane enriched in $\mathrm{PI}(4$, 5)P2 [54] upon VPS4 knockdown [32]. PIs lipids are essential for every aspect of cell division, and especially PI $(4,5)$ P2 lipids play a crucial role in the stabilization of the intercellular bridge just before abscission [74]. In this context, all ESCRT-driven remodeling processes that involve CHMP2B take place at PI(4,5)P2-containing membranes such as HIV-1 budding, plasma membrane repair, cytokinesis, nuclear envelope reformation, and dendritic spine formation $[8,55]$. In vivo, the concentration of PI $(4,5) \mathrm{P} 2$ depends on the membrane type. In our in vitro work, the presence of PI $(4,5) \mathrm{P} 2$ lipids directly affects ESCRT-III recruitment to the membrane. It is probable that the actual concentration of PIP2 in the GUV membrane is lower than in the lipid mixture they were prepared from, as $\mathrm{PI}(4,5) \mathrm{P} 2$ is only partially integrated upon GUV formation and can be solubilized out of the GUV membrane over time [75]. However, our in vitro data suggest that $\mathrm{CHMP} 2 \mathrm{~B}$ recruitment to membranes may be regulated by $\mathrm{PI}(4,5) \mathrm{P} 2$ and thus PIP signaling.

While CHMP2A and CHMP3 assemble homogenously on the GUV membrane, CHMP2B forms a striking reticulum-like structure at the GUV surface at low density. The network co-localizes with $\mathrm{PI}(4,5) \mathrm{P} 2$ indicating clustering of PI(4,5)P2 upon CHMP2B network formation. This clustering of $\mathrm{PI}(4,5) \mathrm{P} 2$ can store elastic stress in the membrane [76]. Moreover, the network formation leads to a strong mechanical stiffening of the membrane. The CHMP2B coat behaves as a rigid shell that can be occasionally fractured upon strong micropipette aspiration. The effect of CHMP3 on CHMP2B membrane binding/polymerization influences also the stiffness of the membrane by softening it compared to CHMP2B only coated membranes. At a smaller scale, on SUVs, this stiffening is also observed. In contrast, the mechanics of GUVs coated with CHMP2A+CHMP3 is almost unchanged. Previous experiments performed on yeast ESCRT-III proteins reported a plastic deformation of membrane coated with Snf7 [30]. As a consequence, the mode of action of ESCRT-III may be regulated by the balance of stiffening and elastic behavior.

In general, in the concentration range explored in our study, with both CHMP2A (+CHMP3) and CHMP2B, we did not observe spontaneous GUV membrane tubulation. Tubulation depends on protein spontaneous curvature, surface fraction, membrane tension, proteinprotein interactions, and protein assembly stiffness [77]. Considering the propensity of the ESCRT-III proteins to form spiral or helical polymers in solution, we could have expected that they might also induce membrane deformation upon polymerization on a lipid membrane. One possible explanation is that we have not included CHMP4, an ESCRT-III member essential for all ESCRTcatalyzed processes [8]. Although CHMP4 assembles on flat membranes [30, 51], it seems to prefer negative membrane curvature for interaction [50]. Thus, the CHMP2B and CHMP2A+CHMP3 membrane binding observed here may have produced assemblies that are different from ESCRT-III assemblies observed in vitro $[78,79]$ lacking spontaneous curvature and/or being too elastic to deform membranes.

The differences observed between CHMP2A and CHMP2B with regard to their membrane interaction and their capacity to affect membrane rigidity indicate that both proteins exert different functions that require different mechanical properties during ESCRT-catalyzed membrane remodeling processes. As an example, the CHMP4B isoform is likely present in the ESCRT-III spirals formed at the mid-body during cytokinesis [80, 81] whereas 
CHMP4C is implicated in abscission control [15]. The increased rigidity imposed by the CHMP2B network might be important for dendritic spine maintenance [82] where it might limit protein diffusion, in agreement with experiments showing that $\mathrm{CHMP2B}$ forms a diffusion barrier at membrane necks reconstituted in vitro [48]. It might also significantly contribute to the mechanical property of the ESCRT-III spirals at the cytokinetic bridge that become very loose when CHMP2B is depleted [65]. Interestingly, CHMP2B function might be modulated by CHMP3, which limits CHMP2B-membrane interaction and softens the CHMP2B assembly. This indicates that in vivo CHMP3 either limits CHMP2B polymerization or/and copolymerizes with CHMP2B into a structure with different mechanical properties, in agreement with observations of copolymerization of ESCRT-III proteins in solution [51].

We thus propose that CHMP3 could play a key regulatory role in the sequence of recruitment of CHMP2B and CHMP2A and in their respective stoichiometry on the membranes during ESCRT-III function. In late steps of cytokinesis, pulling forces exerted by daughter cells on the intercellular bridge appear to regulate abscission, allowing daughter cells to remain connected until they have settled in their final locations. Moreover, counterintuitively, a release of tension conducts membrane scission [83]. Thus, in the case of unmodified membrane softness, ESCRT-III complexes would be able to carry out the membrane scission event. On the contrary, a rigid structure would empede this process. However, a certain degree of membrane rigidity might help the constriction process prior to scission, but at this stage, it is difficult to conclude on this aspect.

\section{Conclusions}

In summary, our data provides evidence that CHMP2A and CHMP2B polymerize differently on membranes and thereby impose different mechanical properties on the membrane structure. Our data thus strongly argue against a sole redundancy of the CHMP2A and CHMP2B proteins and indicate that different isoforms exert complementary functions within the ESCRT-III system.

\section{Methods}

\section{Reagents}

DOPC (1,2-dioleoyl-sn-glycero-3-phosphatidylcholine), DOPS (1,2-dioleoyl-sn-glycero-3-phospho-L-serine), DOPE (1,2-dioleoyl-sn-glycero-3-phosphatidylethanolamine), cholesterol (cholest-5-en-3ß-ol), PI(3)P (1,2-dioleoyl-sn-glycero-3-phospho-(1' -myo-inositol-3' -phosphate)), PI(3,5)P2 (1,2-dioleoyl-sn-glycero-3-phospho(1'-myo-inositol-3',5'-bisphosphate)), PI(4)P (L- $\alpha$-phosphatidylinositol-4-phosphate), PI(4,5)P2 (L- $\alpha$-phosphatidylinositol-4,5-bisphosphate), BODIPY TMR-
PtdIns(4,5)P2, C16 (red PI(4,5) $\mathrm{P}_{2}$ ), 1-oleoyl-2-6-[4(dipyrrometheneboron difluoride) butanoyl] amino hexanoyl-sn-glycero-3-phosphoinositol-4,5-bisphosphate (TopFluor PI $(4,5) \mathrm{P} 2)$, and Egg Rhod PE (L- $\alpha$-phosphatidylethanolamine-N-lissamine rhodamine $B$ sulfonyl) were purchased from Avanti Polar Lipids, Inc. (Avanti Polar Lipids, USA). Stock solutions of lipids were solubilized in chloroform at a concentration of $10 \mathrm{mg} \mathrm{mL}^{-1}$, except for cholesterol and Egg Rho PE dissolved respectively at a concentration of $20 \mathrm{mg} \mathrm{mL}^{-1}$ and $0.5 \mathrm{mg} \mathrm{mL}^{-1}$ and PIPs, which were solubilized in a mixture of chloroform/methanol (70:30) $(\mathrm{v} / \mathrm{v})$ at a concentration of 1 $\mathrm{mg} \mathrm{mL}{ }^{-1}$. All stock solutions were kept under argon and stored at $-20^{\circ} \mathrm{C}$ in amber vials (Sigma-Aldrich, France).

\section{Expression, purification, and labeling of proteins}

Expression and purification of MBP-CHMP2A- $\Delta \mathrm{C}$ (residues 9-161) and CHMP3-FL (residues 1-122) was performed as described in [18]. A final gel filtration chromatography step on a superdex 200 column was performed in a buffer containing $20 \mathrm{mM}$ Hepes $\mathrm{pH} 7.6$, $\mathrm{NaCl} 150 \mathrm{mM}$.

CHMP2B-FL (residues 1-222) and CHMP2B- $\Delta$ C (residues 1-154) were expressed and purified as previously described [32]. Both constructs contain a C-terminal SGSC linker for cysteine-specific labeling. Cells were lysed by sonication in $50 \mathrm{mM}$ Tris $\mathrm{pH} 7.4,1 \mathrm{M} \mathrm{NaCl}, 10$ mM DTT, complete EDTA free, and the soluble fraction was discarded after centrifugation. The pellet was washed three times a buffer containing $50 \mathrm{mM}$ Tris $\mathrm{pH}$ $7.4, \quad 2 \mathrm{M}$ UREA, $2 \%$ Triton X-100, $2 \mathrm{mM} \quad \beta-$ mercaptoethanol, and a final wash in $50 \mathrm{mM}$ Tris $\mathrm{pH}$ 7.4, $2 \mathrm{mM} \beta$-mercaptoethanol. CHMP2B (-FL and $-\Delta \mathrm{C}$ ) was extracted from the pellet using a buffer composed of $50 \mathrm{mM}$ Tris $\mathrm{pH} 7.4,8 \mathrm{M}$ guanidine, $2 \mathrm{mM} \beta$ mercaptoethanol over night at $4{ }^{\circ} \mathrm{C}$. Further purification of solubilized CHMP2B included $\mathrm{Ni}^{2+}$ chromatography in $50 \mathrm{mM}$ Tris $\mathrm{pH} 7.4,8 \mathrm{M}$ urea, refolding by rapid dilution into a buffer containing $50 \mathrm{mM}$ Tris $\mathrm{pH} 7.4,200$ $\mathrm{mM} \mathrm{NaCl}, 2 \mathrm{mM}$ DTT, $50 \mathrm{mM} \mathrm{L}$-glutamate, $50 \mathrm{mM} \mathrm{L}$ arginine at a final concentration of $2 \mu \mathrm{M}$. Refolded CHMP2B was concentrated by $\mathrm{Ni}^{2+}$ chromatography in a buffer containing $50 \mathrm{mM}$ Tris pH 7.4, $200 \mathrm{mM} \mathrm{NaCl}$. A final gel filtration chromatography step was performed on a superdex 75 column in the buffer containing $50 \mathrm{mM}$ Tris $\mathrm{pH}$ 7.4, $100 \mathrm{mM} \mathrm{NaCl}$.

For MBP-CHMP2B- $\Delta$ C production, Escherichia coli BL21 cells were transformed with plasmids and grown at $37{ }^{\circ} \mathrm{C}$ in Luria broth medium to an OD600 of 0.6. Protein expression was induced by the addition of $1 \mathrm{mM}$ arabinose for $3 \mathrm{~h}$ at $37^{\circ} \mathrm{C}$. Cells were harvested by centrifugation and the bacterial pellet was re-suspended in $50 \mathrm{~mL}$ of binding buffer (50 mM Hepes pH 7.6, $300 \mathrm{mM}$ $\mathrm{NaCl}, 300 \mathrm{mM} \mathrm{KCl}$ ). The bacteria were lysed by 
sonication for $5 \mathrm{~min}$ and cell was pelleted by centrifugation at 20,000 rpm for $30 \mathrm{~min}$. The MBP-CHMP2B- $\Delta C$ protein was purified on an amylose affinity column in binding buffer.

Following expression, CHMP proteins were concentrated, labeled overnight at $4{ }^{\circ} \mathrm{C}$ with a ratio of Alexa labeling dye per protein of 2 to 1 . MBP-CHMP2A- $\Delta \mathrm{C}$, CHMP3-FL, and CHMP2B ( $-\triangle \mathrm{C}$ and -FL) were labeled with Alexa 488 succimidyl ester, Alexa 633 succimidyl ester, and Alexa 488 C5 maleimide (Thermo Fisher Scientific), respectively. The excess of free dyes was removed by salt exchange chromatography except for MBP-CHMP2B- $\Delta$ C where a final gel filtration chromatography (superdex 200) step was performed in a buffer containing $50 \mathrm{mM}$ Hepes pH 7.6, $150 \mathrm{mM} \mathrm{NaCl}$. Immediately after labeling, all aliquots were frozen in liquid nitrogen with $0.1 \%$ of methyl cellulose (Sigma-Aldrich) as cryoprotectant. All aliquots were kept at $-80^{\circ} \mathrm{C}$ prior to experiments.

\section{GUV preparation for confocal, spinning disk, and FACS experiments}

GUVs were prepared by spontaneous swelling on polyvinyl alcohol (PVA)-based gels [84]. A thin lipid solution is deposited on a PVA gel (5\% PVA, $50 \mathrm{mM}$ sucrose, 25 $\mathrm{mM} \mathrm{NaCl}$ and $25 \mathrm{mM}$ Tris, at $\mathrm{pH} 7.5)$, dried under vacuum for $20 \mathrm{~min}$ at room temperature and rehydrated with the growth buffer at room temperature. Vesicles form within $45 \mathrm{~min}$ and are extracted by pipetting directly from the slides on top of the PVA gel.

\section{Composition 1}

For confocal and spinning disk microscopy experiments, lipid stock solutions were mixed to obtain DOPC/ DOPS/DOPE/Cholesterol/PI(4,5)P2/PE-Rhodamine

(54.2,10:10:15:10:0.8) (molar ratio) at a concentration of $3 \mathrm{mg} \mathrm{mL} \mathrm{m}^{-1}$ in chloroform. In the following, this GUV composition will be referred to as $10 \%$ PIP2-GUV. In order to detect the PI $(4,5) \mathrm{P} 2$ lipid signal, PE-Rhodamine in the PIP2-GUV lipid stock solution was replaced by TopFluor $\mathrm{PI}(4,5) \mathrm{P}_{2}$ with a molar ratio of $\mathrm{PI}(4,5) \mathrm{P} 2 / \mathrm{Top}$ FluorPI $(4,5)$ P2 of $(8,0.5)$ referred to as FluoPIP2-GUV.

\section{Composition 2}

For FACS microscopy experiments, lipid stock solutions were mixed to obtain DOPC/DOPE/Cholesterol/PI(4, 5)P2/PE-Rhodamine (72.2:10:15:2:0.8) (molar ratio) at a concentration of $3 \mathrm{mg} \mathrm{mL}^{-1}$ in chloroform. In the following, this GUV composition will be referred to as $2 \%$ PIP2-GUV. To compare CHMP protein binding to different PIP species, we replaced PI(4,5)P2 lipids at equal molar ratio by $\mathrm{PI}(3) \mathrm{P}, \mathrm{PI}(4) \mathrm{P}$, and $\mathrm{PI}(3,5) \mathrm{P} 2$ lipids, respectively. In the following, these GUV compositions will be referred to as $2 \% \mathrm{PI}(3) \mathrm{P}-\mathrm{GUV}, 2 \% \mathrm{PI}(4) \mathrm{P}-\mathrm{GUV}$, and $2 \% \mathrm{PI}(3,5) \mathrm{P} 2-\mathrm{GUV}$.

\section{SUV preparation for QCM and AFM experiments}

After preparation of lipid composition 1 , at $3 \mathrm{mg} \mathrm{mL}^{-1}$, in chloroform, the solvent was evaporated by rotating the vial under a gentle stream of nitrogen, at room temperature and then was placed under vacuum for 20 $\mathrm{min}$ at room temperature. The dried lipid film was rehydrated in the appropriate growth buffer solution to obtain a final concentration of $1 \mathrm{mg} \mathrm{mL} \mathrm{m}^{-1}$. The solution was vortexed for $2 \mathrm{~min}$ and then extruded 11 times through a polycarbonate track-etched membrane with pore sizes of $100 \mathrm{~nm}$ [85] or sonicated for 5 min until obtaining a clear colorless solution for small unilamellar vesicle (SUV) formation. Produced SUVs were either used freshly for QCM-D experiments and for HS-AFM indentation experiments or stored at $-20^{\circ} \mathrm{C}$ in amber vials (Sigma-Aldrich, France) for further use. In the following, this SUV composition will be referred to as $10 \%$ PIP2-SUV.

To compare CHMP2B protein binding in the absence of $\mathrm{PI}(4,5) \mathrm{P} 2$ and to increase the net negative charge of the membrane for QCM-D experiments, SUVs were produced containing DOPC/DOPS/DOPE/Cholesterol/ PE-Rhodamine (44.2:30:10:15:0.8) (molar ratio) or (34.2: 40:10:15:0.8), at a concentration of $3 \mathrm{mg} \mathrm{mL}^{-1}$ in chloroform referred to as 30\% DOPS-SUV and 40\% DOPSSUV, respectively. Moreover, to compare CHMP2B protein binding to a membrane incorporating a higher amount of negative charges as well as PIP lipids, we replaced the $10 \%$ molar ratio of $\mathrm{PI}(4,5) \mathrm{P} 2$ in the PIP2-SUV by $10 \%$ molar ratio of PI(3-5)P3 lipids. In all QCM-D experiments, quartz crystal resonance frequency shifts were measured at the overtone 5 of the oscillating crystal and therefore defined as $\Delta \vartheta_{5}$.

\section{CHMP supramolecular assembly on GUVs observed by fluorescence microscopy}

Freshly produced 10\% PIP2-GUVs were incubated with CHMP proteins at concentrations ranging from $50 \mathrm{nM}$ to $2 \mu \mathrm{M}$ in BP buffer (Tris $25 \mathrm{Mm}, \mathrm{NaCl} 50 \mathrm{mM} \mathrm{pH} 7.5$ ) in isotonic conditions for 15 to $30 \mathrm{~min}$. Then, CHMPcoated GUVs were diluted 20 times and transferred to the observation chamber, previously passivated with the $\beta$-casein solution and rinsed twice with BP buffer.

Supramolecular assembly of CHMP proteins on GUVs was visualized on an inverted Spinning Disk Confocal Roper/Nikon. The spinning disk is equipped with the camera, EMCCD $512 \times 512$ Andor Technology (pixel size $16 \mu \mathrm{m})$, an objective ( $\times 100$ CFI Plan Apo VCoil NA 1,4 WD 0,13), and 3 lasers (491, 561, $633 \mathrm{~nm} 100 \mathrm{~mW}$ ). The exposure time for all images was $50 \mathrm{~ms}$. 
To further characterize and compare the interaction of CHMP proteins on GUVs, we measured the total intensity of the protein on the vesicle and normalized this value by the GUV area.

Image acquisition for protein quantification was performed using a confocal microscope composed of an inverted microscope (Eclipse TE2000 from Nikon), two objectives ( $\times 60$ water immersion and $\times 100$ oil immersion), a $\mathrm{C} 1$ confocal head from Nikon, three lasers $(\lambda=488 \mathrm{~nm}, \lambda=561 \mathrm{~nm}$, and $\lambda=633 \mathrm{~nm})$. One confocal plane image was taken for each set tension.

\section{FACS experiment for protein-lipid binding assay}

2\% PI-GUV and CHMP fluorescence intensity was measured with a BD LSRFORTESSA flow cytometry instrument. Data analysis was performed with BD FACS Diva software.

The collected GUVs were transferred to BP buffer and incubated $30 \mathrm{~min}$ with CHMP proteins at $500 \mathrm{nM}$. The vesicle concentration was adjusted in order to count about 10,000 events per condition every $60 \mathrm{~s}$ at high speed.

2\% PI-GUVs were labeled with Egg Rhod PE $(0.8 \%$ w/ w), CHMP2B labeled with Alexa 488, CHMP2A labeled with Alexa 488, and CHMP3 labeled with Alexa 633. Alexa 488 was excited with a 488-nm laser, and the emission was detected through a 530/30 standard bandpass filter. Alexa 633 was excited with a 633-nm laser, and the emission was detected through a $670 / 30$ bandpass filter. Egg Rhod PE was excited with a 532-nm laser, and the emission was detected through a $610 / 20$ bandpass filter. Two signals were closely analyzed: the protein fluorescent signal and the lipid fluorescent signal. Thus, the fluorescence intensity of the membrane and the fluorescence intensity of the proteins are respectively proportional to the amount of fluorophores in the vesicle and proteins bound to it or present in the detection zone and unbound. The intensity plot displaying the protein fluorescence signal as a function of the lipid fluorescent signal presents 3 regions: (i) unbound proteins (single-positive for proteins only in the top left quadrant), (ii) CHMP proteins bound to GUVs (doublepositive for proteins and lipids in the top right quadrant), and (iii) GUVs free of proteins (single-positive for lipids only in the lower right quadrant).

\section{QCM-D experiments}

Supported lipid bilayers (SLBs) were generated with or without PIP lipids. In the absence of PI $(4,5)$ P2, SLB made of $30 \%$ and $40 \%$ DOPS-SUV composition were produced with a buffer containing $\mathrm{Ca}^{2+}(150 \mathrm{mM} \mathrm{NaCl}$, $10 \mathrm{mM}$ Tris (at $\mathrm{pH} 7.5)+2 \mathrm{mM} \mathrm{Ca}^{2+}$ ) [41]. After SLB formation, the bilayer was rinsed with the same buffer but supplemented with EDTA $(150 \mathrm{mM} \mathrm{NaCl}, 10 \mathrm{mM}$
Tris $\mathrm{pH} 7.5,10 \mathrm{mM}$ EDTA) to remove $\mathrm{Ca}^{2+}$ excess. SLBs were also produced in the presence of PIP lipids (PI(4, 5)P2 or PI(3-5)P3), with PIP2-SUV or PIP3-SUV lipid compositions, respectively. SLB formation was achieved in a buffer containing $150 \mathrm{mM} \mathrm{KCl}, 20 \mathrm{mM}$ citrate $\mathrm{pH}=$ 4.8 [42]. Following SLB formation, CHMP proteins were injected at a concentration of $200 \mathrm{nM}$ in BP buffer. The interaction between the proteins and the lipid bilayer was directly measured from the fifth overtone of the frequency shift $\left(\Delta 9_{5}\right)$.

QCM-D measurements were performed using a QSense E4 system ( $Q$ sense; Gothenburg, Sweden). The mass sensor is a silicon dioxide-coated quartz crystal microbalance $\mathrm{SiO}_{2}$ (QSX-303 Lot Quantum Design France) with a fundamental frequency of $4.95 \mathrm{MHz}$. The liquid flow was controlled using a high-precision multichannel dispenser (IPC; ISMATEC-Germany). All experiments were performed at room temperature with a flow rate of $50 \mu \mathrm{L} \mathrm{min}{ }^{-1}$.

\section{Micropipette experiments}

The experimental chamber and the micropipette made of a borosilicate capillary (1-mm outer diameter and 0.58-mm inner diameter (Harvard Apparatus, UK)) introduced into the chamber are passivated with a $\beta$ casein solution at $5 \mathrm{mg} \mathrm{mL}^{-1}$ in sucrose $25 \mathrm{mM}, \mathrm{NaCl}$ $50 \mathrm{mM}$, and Tris $25 \mathrm{mM}$ (pH 7.5) for $15 \mathrm{~min}$. The chamber is rinsed twice with BP buffer. Then, PIP2-GUVs pre-incubated with CHMP proteins are added to the chamber. Once the chamber is sealed with mineral oil, the zero pressure is measured and the aspiration assay can begin by decreasing the water height gradually, thus increasing the applied tension on the vesicle.

The explored tensions for the aspiration experiments with the different CHMP proteins range up to 1.6 $\mathrm{mN} \mathrm{m}{ }^{-1}$ (corresponding to the membrane enthalpic regime). The software EZ-C1 was used for the acquisition of the confocal images.

At high tension, in the enthalpic regime, an apparent elastic stretching modulus of the membrane $X$ can be deduced from the linear variation of the fractional excess area $\Delta \alpha\left(\Delta \alpha=\pi D_{p}\left(1-D_{p} / D_{v}\right) \Delta L_{p} / A_{0}\right.$ where $\Delta L_{p}$ is the variation of the tongue length and $A_{0}$ the initial area of the GUV) as a function of the applied tension $\sigma$ using $\Delta$ $\alpha=\Delta \alpha_{0}+\frac{1}{x} \sigma$ [86], with $\Delta \alpha_{0}$ being the initial excess area for the reference tension $\sigma_{0}$. According to the YoungLaplace equation, the membrane tension is equal to $\sigma$ $=\Delta P \times R_{p} /\left(2 \times\left(1-\frac{R_{p}}{R_{v}}\right)\right)$ where $\Delta P$ is the difference of pressure between the interior of the micropipette and the chamber and $R_{p}$ and $R_{v}$ are respectively the pipette and vesicle radius [63]. 


\section{Osmotic shock on GUVs}

$10 \%$ PIP2-GUVs were either co-incubated with $500 \mathrm{nM}$ CHMP2B- $\Delta \mathrm{C}$ in $50 \mathrm{mM} \mathrm{NaCl}$ and $25 \mathrm{mM}$ Tris, at $\mathrm{pH}$ 7.4 buffer (CHMP protein binding buffer referred as BP buffer) or transferred to the same buffer free of protein (osmolarity equal to $125 \mathrm{mOsm} \mathrm{L}^{-1}$ ). CHMP2B-coated GUVs and CHMP2B-free GUVs were then transferred to a hyperosmotic buffer with increasing sodium chloride concentrations up to $250 \mathrm{mM} \mathrm{NaCl}$. The effect of the osmotic shock was visualized using confocal microscopy.

\section{HS-AFM imaging-based deformation experiment}

PIP2-SUVs were immobilized on a freshly cleaved mica surface and placed into the AFM chamber with BP buffer. For studying the vesicles with proteins, prior to immobilization to the surface, the PIP2-SUVs were pre-incubated with either $1 \mu \mathrm{M}$ of CHMP2B or $1 \mu \mathrm{M}$ of $\mathrm{CHMP} 2 \mathrm{~B}+2 \mu \mathrm{M}$ of CHMP3 or $1 \mu \mathrm{M}$ of CHMP2A $+2 \mu \mathrm{M}$ of CHMP3 for 30 min to allow full protein coverage on the SUV surface. A high-speed amplitude modulation tapping mode AFM (RIBM, Japan) was used for imaging [87-89] and deformation experiments, with ultra-short cantilevers (spring constant $0.15 \mathrm{~N} / \mathrm{m}$, Nanoworld). Initial imaging (at minimum force) was performed at a free cantilever oscillation amplitude of $5.4 \mathrm{~nm}$ and a set-point amplitude at $4.3 \mathrm{~nm}$. The imaging rate was 0.5 frame/s. We regulated the set-point amplitude in a stepwise manner, while keeping the free amplitude constant, in order to increase the imaging force. The imaging force can be estimated in the first approximation as $F=k \Delta z$, where $k$ is the spring constant of the cantilever and $\Delta z$ is the difference between free and setpoint amplitude of the cantilever oscillation. It follows that the images were acquired with an estimated minimal force of $\sim 150 \mathrm{pN}$. For the measurement of membrane mechanics with and without proteins, image acquisition was first performed at minimal force $(\sim 150 \mathrm{pN})$. Next, step by step, the imaging force was increased with $9 \%$ increments, by decreasing the set-point amplitude. After reaching the maximal force, after $\sim 8$ steps and an estimated final imaging force of $\sim 270 \mathrm{pN}$, the tapping force was reduced again to its lowest value $\quad(\sim 150 \mathrm{pN})$, and the height recovery was recorded. Only those vesicles that exhibited a height recovery of at least $90 \%$ of their initial height were considered to be elastically deformed and were included in the analysis. Errors in the relative stiffness are given as standard error of the mean (SEM). Images were analyzed using IgorPro scripts of the AFM manufacturer (RIBM) and ImageJ scripts.

\section{Supplementary Information}

The online version contains supplementary material available at https://doi. org/10.1186/s12915-021-00983-9.

Additional file 1: Figure S1. Evolution of the ESCRT-III complex. (A) Table illustrating the ESCRT-III complex function, origin and homologs in S. cerevisiae and $H$. sapiens. (B) Distribution of Vps 2 and $V p s 24$ genes across Eukaryotes showing the presence of two Vps2 genes in high organisms. (C) Table illustrating the implication of ESCRT-III subunits in different subcellular locations in S. cerevisiae and H. sapiens. The names are the human homologs in case of $\mathrm{S}$. cerevisiae.

Additional file 2: Figure S2. Study of CHMP protein-membrane interaction. (A) Optimization of the buffer conditions to optimize the binding of CHMP2B- $\triangle$ C (noted here CHMP2B) at $500 \mathrm{nM}$. Pre-formed vesicles were incubated with CHMP2B- $\triangle \mathrm{C}$ in buffers with different salt concentrations ranging from $0 \mathrm{mM}$ to $100 \mathrm{mM} \mathrm{NaCl}$ (+Tris $25 \mathrm{mM}$ at pH 7.5) and imaged with confocal microscope after 30 min incubation. Lipid signal is shown in magenta and protein signal in green. Scale bar: $5 \mu \mathrm{m}$. (B) Confocal image of MBPCHMP2A- $\triangle C$ (noted here CHMP2A) without TEV (Top line) and in the presence of TEV to cleave the MBP tag (Bottom line). Saturated protein fluorescent signal is represented in yellow. Cleavage of MBP tag slightly increases the interaction but induces aggregation. Scale bar: $30 \mu \mathrm{m}$. (C) Spinning disk image of GUV incubated with MBP-CHMP2B- $\triangle C$ (noted here MBP-CHMP2B) at a concentration of $50 \mathrm{nM}$ (left image, scale bar $=10 \mu \mathrm{m}$ ) and at $200 \mathrm{~nm}$ (right image; scale bar $=5 \mu \mathrm{m}$ ). (D) Confocal images of GUV incubated with MBP-CHMP2B- $\triangle$ C at a concentration of $1 \mu \mathrm{M}$ (noted here MBP-CHMP2B), submitted to an osmotic pressure difference equal to 150\% (Osmolarity inside and outside the GUV are respectively $120 \mathrm{mOsm} \cdot \mathrm{L}^{-1}$ and $315 \mathrm{mOsm} \cdot \mathrm{L}^{-1}$ ). Scale bar: $10 \mu \mathrm{m}$.

Additional file 3: Figure S3. (A) Histograms of $C H M P 2 B-\Delta C$ protein fluorescence intensity for PI(3)P, PI(4)P, PI(3,5)P2 and PI(4,5)P2 GUVs (lipid composition 2). (B) Comparison of the binding density of MBP-CHMP2A$\triangle C+C H M P 3$ and of CHMP2B- $\Delta C$ to GUVs with different charged lipids, measured by FACS, corresponding to Fig. 1d. The values are normalized to their respective binding density to DOPS. ${ }^{* *}=p$-value $<0.01$ (Student's t-test). $N=4$ (number of FACS experiment with about $10^{4}$ counted events per experiment, per condition). (C) QCM-D experiment displaying the typical frequency shift of $-25 \mathrm{~Hz}$ after supported bilayer formation and a frequency shift $\Delta \mathrm{U}_{5}$ representative of the amount of protein bound to the bilayer. (D) Spinning disk images of interaction of MBP-CHMP2A$\Delta \mathrm{C}+\mathrm{CHMP3}$ in BP buffer on $10 \% \mathrm{PI}(4,5) \mathrm{P} 2$-containing GUVs. CHMP2A- $\Delta \mathrm{C}$ fluorescent signal is displayed. A z-projection is represented. The different panels corresponding to 3 representative GUVS show the homogeneous coverage of the co-polymer as a function of protein concentration and incubation time. First panel: CHMP2A and CHMP3 are incubated at 500 $\mathrm{nM}$ and $2 \mu \mathrm{M}$, respectively, for $15 \mathrm{~min}$. Second panel: CHMP2A and CHMP3 are incubated at $1 \mu \mathrm{M}$ and $4 \mu \mathrm{M}$, respectively, for 15 min. Third panel: CHMP2A and CHMP3 are incubated at $500 \mathrm{nM}$ and $2 \mu \mathrm{M}$, respectively, for $60 \mathrm{~min}$. Scale bar, $10 \mu \mathrm{m}$.

Additional file 4: Figure S4. Deformation of bare vesicles and vesicles covered with CHMP2A + CHMP3 or CHMP2B + CHMP3. HS-AFM images of a vesicle covered with CHMP2A and CHMP 3 (left column) and a vesicle covered with $\mathrm{CHMP} 2 \mathrm{~B}$ and $\mathrm{CHMP3}$ proteins (right column). The deformability of the SUVS coated with corresponding proteins upon increased applied force are shown at intermediate force increments of $27 \%$ (second panels) and at higher force increment, 81\% (third panels).

Additional file 5: Movie S1. Typical example of vesicle response upon increasing and decreasing imaging force. It can be observed that the vesicle restores its height after the final decrease in imaging force.

Additional file 6: Figure S5. Deformation of bare vesicles and vesicles covered with CHMP proteins. (A) Reduction of vesicle height under increasing force for bare vesicles. 'Zero' force increment represents the minimum imaging force ( $150 \mathrm{pN}$ ). (B) Example of deformation for a $\sim 60 \mathrm{~nm}$ vesicle over increasing force up to $80 \%$ of the initial imaging force. (C) Represents the transformation of vesicle height to relative height for each point for the curve in $\mathbf{D}$. (D) represents the relative height vs force increment for all the curves from panel $\mathbf{A}$ for bare SUVS. (E) represents the relative height vs force increment for SUV covered with CHMP2B (left), CHMP2B + CHMP3 (middle) and CHMP2A + CHMP3 (right). 
Additional file 7: Figures S6. Test of mutual interaction between CHMP2B and CHMP3 in solution. CHMP2B- $\triangle$ C (first gel) and CHMP3-FL (second gel) in Hepes Buffer have been deposited on a sucrose gradient. $100 \mu \mathrm{L}$ of CHMP2B- $\Delta \mathrm{C}$ at $10 \mu \mathrm{M}$ has been incubated with $100 \mu \mathrm{l}$ CHMP3FL at $10 \mu \mathrm{M}$ and deposit on a sucrose gradient (third gel). No aggregation is observed on the bottom of the gradient. The presence of CHMP3 does not induce CHMP2B aggregation.

\section{Acknowledgements}

The authors would like to thank John Manzi for the help in the biochemistry characterization of the ESCRT complexes.

\section{Authors' contributions}

N.M. performed the expression, purification, and labeling of the proteins. M.A. and N.D.F. performed the GUV preparation, the confocal imaging, the FACS, and the micropipette experiments. M.A. and S. Mai prepared the SUV for QCM and AFM experiments. M.A. and M.B. performed the QCM-D experiments. S.Mai. performed the AFM experiments. Experiments have been designed and discussed by W.W., W.R., P.B., and S.Man. M.A., W.W., P.B., and S.Man. wrote the manuscript. The results and their interpretation were discussed by all of the authors. All authors read and approved the final manuscript.

\section{Funding}

We acknowledge support from FINOVI (WW), the ANR (ANR-14-CE09-000301) (WW, PB), the Institut Universitaire de France (IUF; WW), sorbonne Université (Grant Emergence; S.Man) and from the platforms of the Grenoble Instruct-ERIC center (ISBG; UMS 3518 CNRS-CEA-UGA-EMBL) within the Grenoble Partnership for Structural Biology (PSB). Platform access was supported by FRISBI (ANR-10-INBS-05-02) and GRAL, a project of the University Grenoble Alpes graduate school (Ecole Universitaire de Recherche) CBH-EUR-GS (ANR17-EURE-0003). We further acknowledge funding through a NWO VIDI grant (WHR) and an EU Marie Curie fellowship (INTERACT 751404), (S.Mai.). P.B. group greatly acknowledges the Nikon Imaging Center (Institut Curie, Paris), a member of the France Biolmaging national research infrastructure (ANR-10INSB-04) as well as the flow Cytometry Platform of the Institute Curie for technical support in microscopy and flow cytometry respectively. M.A. was funded by the Université Pierre et Marie Curie/sorbonne Université, Doctoral school "Physique en lle de France" (ED-564) and the Fondation pour la Recherche Médicale. N.D.F was funded by post-doc fellowships from the Institute Curie, the Fondation pour la Recherche Médicale a EMBO nonstipendiary long-term fellowship (ALTF 818-2016) and the European Union's Horizon 2020 research and innovation program (MSCA No. 751715). P.B. group belongs to the CNRS consortium CellTiss, to the Labex CelTisPhyBio (ANR-11- 798 LABX0038), and to Paris Sciences et Lettres (ANR-10-IDEX-000102).

\section{Availability of data and materials}

The authors confirm that the data supporting the findings of this study are available within the article and its supplementary materials.

\section{Declarations}

\section{Ethics approval and consent to participate}

Not applicable.

\section{Consent for publication}

Not applicable.

\section{Competing interests}

The authors declare that they have no competing interests.

\section{Author details}

${ }^{1}$ Laboratoire Physico Chimie Curie, Institut Curie, Université PSL, Sorbonne Université, CNRS UMR168, 75005 Paris, France. ${ }^{2}$ Moleculaire Biofysica, Zernike Instituut, Rijksuniversiteit Groningen, Nijenborgh 4, 9747 AG Groningen, The Netherlands. ${ }^{3}$ Univ. Grenoble Alpes, CNRS, CEA, Institut de Biologie Structurale (IBS), 38000 Grenoble, France. ${ }^{4}$ Umeå University, Department of Clinical Microbiology \& Wallenberg Centre for Molecular Medicine, 90185 Umeå, Sweden.
Received: 23 January 2021 Accepted: 16 February 2021

Published online: 08 April 2021

\section{References}

1. Hurley JH. ESCRTs are everywhere. EMBO J. 2015;34:2398-407.

2. Henne WM, Stenmark H, Emr SD. Molecular mechanisms of the membrane sculpting ESCRT pathway. Cold Spring Harb Perspect Biol. 2013;5:a016766.

3. Scheffer LL, et al. Mechanism of Ca2+-triggered ESCRT assembly and regulation of cell membrane repair. Nat Commun. 2014;5:5646.

4. Loncle N, Agromayor M, Martin-Serrano J, Williams DW. An ESCRT module is required for neuron pruning. Sci Rep. 2015;5:8461.

5. Sadoul $R$, et al. The role of ESCRT during development and functioning of the nervous system. Semin Cell Dev Biol. 2018;74:40-9.

6. Olmos Y, Hodgson L, Mantell J, Verkade P, Carlton JG. ESCRT-III controls nuclear envelope reformation. Nature. 2015;522:236.

7. Vietri M, et al. Spastin and ESCRT-III coordinate mitotic spindle disassembly and nuclear envelope sealing. Nature. 2015;522:231.

8. Scourfield EJ, Martin-Serrano J. Growing functions of the ESCRT machinery in cell biology and viral replication. Biochem Soc Trans. 2017:45:613-34

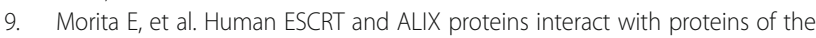
midbody and function in cytokinesis. EMBO J. 2007;26:4215-27.

10. Weiss ER, Göttlinger $H$. The role of cellular factors in promoting HIV budding. J Mol Biol. 2011;410(4):525-33.

11. Leung KF, Dacks JB, Field MC. Evolution of the multivesicular body ESCRT machinery; retention across the eukaryotic lineage. Traffic. 2008;9:1698-716.

12. Teis D, Saksena S, Emr SD. Ordered assembly of the ESCRT-III complex on endosomes is required to sequester cargo during MVB formation. Dev Cell. 2008;15(4):578-89.

13. Carlton JG, Agromayor M, Martin-Serrano J. Differential requirements for Alix and ESCRT-III in cytokinesis and HIV-1 release. Proc Natl Acad Sci U S A. 2008;105:10541-6.

14. Morita E, Sandrin V, McCullough J, Katsuyama A, Baci Hamilton I, Sundquist WI. ESCRT-III protein requirements for HIV-1 budding. Cell Host Microbe. 2011:9:235-42.

15. Carlton JG, Caballe A, Agromayor M, Kloc M, Martin-Serrano J. ESCRT-III governs the Aurora B-mediated abscission checkpoint through CHMP4C. Science. 2012;336:220-5.

16. Jimenez AJ, Maiuri P, Lafaurie-Janvore J, Divoux S, Piel M, Perez F. ESCRT machinery is required for plasma membrane repair. Science. 2014;343: 1247136.

17. Olmos Y, Carlton JG. The ESCRT machinery: new roles at new holes. Curr Opin Cell Biol. 2016:38:1-11.

18. Muziol T, et al. Structural basis for budding by the ESCRT-III factor CHMP3. Dev Cell. 2006;10:821-30

19. Bajorek M, et al. Structural basis for ESCRT-III protein autoinhibition. Nat Struct Mol Biol. 2009;16:754-62.

20. Xiao J, Chen XW, Davies BA, Saltiel AR, Katzmann DJ, Xu Z. Structural basis of Ist1 function and Ist1-Did2 interaction in the multivesicular body pathway and cytokinesis. Mol Biol Cell. 2009;20:3514-24.

21. Zamborlini A, Usami Y, Radoshitzky SR, Popova E, Palu G, Gottlinger H. Release of autoinhibition converts ESCRT-III components into potent inhibitors of HIV-1 budding. Proc Natl Acad Sci U S A. 2006;103:19140-5.

22. Shim S, Kimpler LA, Hanson PI. Structure/function analysis of four core ESCR T-III proteins reveals common regulatory role for extreme C-terminal domain. Traffic. 2007:8:1068-79.

23. Lata $S$, et al. Structural basis for autoinhibition of ESCRT-III CHMP3. J Mol Biol. 2008;378:818-27.

24. Ghazi-Tabatabai S, et al. Structure and disassembly of filaments formed by the ESCRT-III subunit Vps24. Structure. 2008;16:1345-56.

25. Lata $\mathrm{S}$, et al. Helical structures of ESCRT-III are disassembled by VPS4. Science. 2008;321:1354-7.

26. Henne WM, Buchkovich NJ, Zhao Y, Emr SD. The endosomal sorting complex ESCRT-II mediates the assembly and architecture of ESCRT-III helices. Cell. 2012;151:356-71.

27. Shen QT, et al. Structural analysis and modeling reveals new mechanisms governing ESCRT-III spiral filament assembly. J Cell Biol. 2014;206:763-77.

28. Dobro MJ, et al. Electron cryotomography of ESCRT assemblies and dividing Sulfolobus cells suggests that spiraling filaments are involved in membrane scission. Mol Biol Cell. 2013;24:2319-27. 
29. Moriscot C, et al. Crenarchaeal CdvA forms double-helical filaments containing DNA and interacts with ESCRT-III-like CdvB. PLoS One. 2011;6: e21921.

30. Chiaruttini N, et al. Relaxation of loaded ESCRT-III spiral springs drives membrane deformation. Cell. 2015;163:866-79.

31. Hanson PI, Roth R, Lin Y, Heuser JE. Plasma membrane deformation by circular arrays of ESCRT-III protein filaments. J Cell Biol. 2008;180(2):389-402.

32. Bodon $\mathrm{G}$, et al. Charged multivesicular body protein 2B (CHMP2B) of the endosomal sorting complex required for transport-III (ESCRT-III) polymerizes into helical structures deforming the plasma membrane. J Biol Chem. 2011; 286:40276-86

33. Cashikar AG, Shim S, Roth R, Maldazys MR, Heuser JE, Hanson PI. Structure of cellular ESCRT-III spirals and their relationship to HIV budding. Elife. 2014; 3:e02184.

34. Guizetti J, et al. Cortical constriction during abscission involves helices of ESCRT-III-dependent filaments. Science. 2011;331(6024):1616-20.

35. McCullough J, et al. Structure and membrane remodeling activity of ESCRTIII helical polymers. Science. 2015;350:1548-51.

36. Lata S, Schoehn G, Solomons J, Pires R, Gottlinger HG, Weissenhorn W. Structure and function of ESCRT-III. Biochem Soc Trans. 2009;37:156-60.

37. Tang $S$, et al. Structural basis for activation, assembly and membrane binding of ESCRT-III Snf7 filaments. Elife. 2015;4:e12548.

38. Pires $R$, et al. A crescent-shaped ALIX dimer targets ESCRT-III CHMP4 filaments. Structure. 2009;17:843-56.

39. Pfitzner A-K, et al. An ESCRT-III polymerization sequence drives membrane deformation and fission. Cell. 2020;182:1140-55.e1118.

40. Huber ST, Mostafavi S, Mortensen SA, Sachse C. Structure and assembly of ESCRT-III helical Vps24 filaments. Science Advances. 2020;6:eaba4897.

41. Bertin A, et al. Human ESCRT-III polymers assemble on positively curved membranes and induce helical membrane tube formation. Nat Commun. 2020;11:2663.

42. Nguyen $\mathrm{HC}$, et al. Membrane constriction and thinning by sequential ESCR T-III polymerization. Nat Struct Mol Biol. 2020;27:392-9.

43. Weissenhorn W, Poudevigne E, Effantin G, Bassereau P. How to get out: ssRNA enveloped viruses and membrane fission. Current Opinion Virol. 2013;3:159-67.

44. Effantin G, et al. ESCRT-III CHMP2A and CHMP3 form variable helical polymers in vitro and act synergistically during HIV-1 budding. Cell Microbiol. 2013;15:213-26.

45. Morita E, Colf LA, Karren MA, Sandrin V, Rodesch CK, Sundquist WI. Human ESCRT-III and VPS4 proteins are required for centrosome and spindle maintenance. Proc Natl Acad Sci U S A. 2010;107:12889-94.

46. Sezgin E, Schwille P. Model membrane platforms to study proteinmembrane interactions. Mol Membr Biol. 2012;29:144-54.

47. Lagny TJ, Bassereau P. Bioinspired membrane-based systems for a physical approach of cell organization and dynamics: usefulness and limitations. Interface Focus. 2015;5:20150038.

48. De Franceschi N, et al. The ESCRT protein CHMP2B acts as a diffusion barrier on reconstituted membrane necks. J Cell Sci. 2019;132:jcs217968.

49. Fyfe I, Schuh AL, Edwardson JM, Audhya A. Association of ESCRT-II with VPS20 generates a curvature sensitive protein complex capable of nucleating filaments of ESCRT-III. J Biol Chem. 2011;286:34262-70.

50. Lee $\mathrm{HH}$, Kai H, Carlson LA, Groves JT, Hurley JH. Negative membrane curvature catalyzes nucleation of endosomal sorting complex required for transport (ESCRT)-III assembly. Proc Natl Acad Sci U S A. 2015;112:15892-7.

51. Mierzwa BE, et al. Dynamic subunit turnover in ESCRT-III assemblies is regulated by Vps4 to mediate membrane remodelling during cytokinesis. Nat Cell Biol. 2017;19:787-98.

52. Schöneberg J, et al. ATP-dependent force generation and membrane scission by ESCRT-III and Vps4. Science. 2018;362:1423-8.

53. Wollert T, Hurley JH. Molecular mechanism of multivesicular body biogenesis by ESCRT complexes. Nature. 2010;464(7290):864-9.

54. Di Paolo G, De Camilli P. Phosphoinositides in cell regulation and membrane dynamics. Nature. 2006;443:651.

55. Garnier-Lhomme M, et al. Nuclear envelope remnants: fluid membranes enriched in STEROLS and polyphosphoinositides. PLOS One. 2009;4:e4255.

56. Lamblet $M$, Delord $B$, Johannes $L$, van Effenterre D, Bassereau P. Key role of receptor density in colloid/cell specific interaction: a quantitative biomimetic study on giant vesicles. Eur Physical J E. 2008;26:205.

57. McLaughlin S, Wang J, Gambhir A, Murray D. PIP2 and proteins: interactions, organization, and information flow. Annu Rev Biophys Biomol Struct. 2002; 31:151-75.
58. Baumann MK, Amstad E, Mashaghi A, Textor M, Reimhult E. Characterization of supported lipid bilayers incorporating the phosphoinositides phosphatidylinositol 4,5-biphosphate and phosphoinositol-3,4,5triphosphate by complementary techniques. Biointerphases. 2010;5:114-9.

59. van den Bogaart $G$, ter Beest M. Domains of phosphoinositides in the plasma membrane. In: Cambi A, Lidke DS, editors. Cell membrane nanodomains. Boca Raton: CRC Press; 2014

60. Raab M, et al. ESCRT III repairs nuclear envelope ruptures during cell migration to limit DNA damage and cell death. Science. 2016;352:359-62.

61. Denais $C M$, et al. Nuclear envelope rupture and repair during cancer cell migration. Science. 2016;352:353-8.

62. Moser von Filseck J, et al. Anisotropic ESCRT-III architecture governs helical membrane tube formation. Nature Communications. 2020;11:1516.

63. Kwok R, Evans E. Thermoelasticity of large lecithin bilayer vesicles. Biophys J. 1981:35:637-52

64. Rawicz W, Olbrich KC, McIntosh T, Needham D, Evans E. Effect of chain length and unsaturation on elasticity of lipid bilayers. Biophys J. 2000;79: 328-39.

65. Bernard A-L, Guedeau-Boudeville M-A, Jullien L, di Meglio J-M. Raspberry vesicles. Biochim Biophys Acta Biomembr. 2002;1567:1-5.

66. Babst M, Katzmann DJ, Estepa-Sabal EJ, Meerloo T, Emr SD. ESCRT-III: an endosome-associated heterooligomeric protein complex required for MVB sorting. Dev Cell. 2002;3:271-82.

67. Martinelli N, et al. CC2D1A is a regulator of ESCRT-III CHMP4B. J Mol Biol. 2012:419:75-88.

68. Ventimiglia LN, et al. CC2D1B coordinates ESCRT-III activity during the mitotic reformation of the nuclear envelope. Developmental Cell. 2018;47: 547-63.e546.

69. McMillan BJ, Tibbe C, Drabek AA, Seegar TCM, Blacklow SC, Klein T. Structural basis for regulation of ESCRT-III complexes by Lgd. Cell Rep. 2017; 19:1750-7.

70. Jouvenet $\mathrm{N}$, Zhadina M, Bieniasz PD, Simon SM. Dynamics of ESCRT protein recruitment during retroviral assembly. Nat Cell Biol. 2011;13:394-401.

71. Baumgartel $V$, et al. Live-cell visualization of dynamics of HIV budding site interactions with an ESCRT component. Nat Cell Biol. 2011;13:469-74.

72. Prescher J, et al. Super-resolution imaging of ESCRT-proteins at HIV-1 assembly sites. PLoS Pathog. 2015;11:e1004677.

73. Johnson DS, Bleck M, Simon SM. Timing of ESCRT-III protein recruitment and membrane scission during HIV-1 assembly. eLife. 2018;7:e36221.

74. Cauvin C, Echard A. Phosphoinositides: lipids with informative heads and mastermind functions in cell division. Biochim Biophys Acta. 2015; 1851:832-43.

75. Beber $\mathrm{A}$, et al. Septin-based readout of $\mathrm{PI}(4,5) \mathrm{P} 2$ incorporation into membranes of giant unilamellar vesicles. Cytoskeleton. 2019;76:92-103.

76. Mulet $\mathrm{X}$, Templer RH, Woscholski R, Ces O. Evidence that phosphatidylinositol promotes curved membrane interfaces. Langmuir. 2008:24:8443-7.

77. Shi Z, Baumgart T. Membrane tension and peripheral protein density mediate membrane shape transitions. Nat Commun. 2015;6:5974.

78. Peel S, Macheboeuf P, Martinelli N, Weissenhorn W. Divergent pathways lead to ESCRT-III catalyzed membrane fission. Trends Biochem Sci. 2011;36: 199-210.

79. McCullough J, Frost A, Sundquist Wl. Structures, functions, and dynamics of ESCRT-III/Nps4 membrane remodeling and fission complexes. Annu Rev Cell Dev Biol. 2018;34, null.

80. Elia N, Sougrat R, Spurlin TA, Hurley JH, Lippincott-Schwartz J. Dynamics of endosomal sorting complex required for transport (ESCRT) machinery during cytokinesis and its role in abscission. Proc Natl Acad Sci U S A. 2011; 108:4846-51.

81. Goliand I, et al. Resolving ESCRT-III spirals at the intercellular bridge of dividing cells using 3D STORM. Cell Rep. 2018;24:1756-64.

82. Chassefeyre $R$, et al. Regulation of postsynaptic function by the dementiarelated ESCRT-III subunit CHMP2B. J Neurosci. 2015;35:3155-73.

83. Lafaurie-Janvore J, et al. ESCRT-III assembly and cytokinetic abscission are induced by tension release in the intercellular bridge. Science. 2013;339: 1625-9.

84. Weinberger A, et al. Gel-assisted formation of giant unilamellar vesicles. Biophys J. 2013;105:154-64.

85. Méléard P, Bagatolli LA, Pott T. Chapter 9 - giant unilamellar vesicle electroformation: from lipid mixtures to native membranes under physiological conditions. Methods Enzymol. 2009;465:161-76. 
86. Evans E, Rawicz W. Entropy-driven tension and bending elasticity in condensed-fluid membranes. Phys Rev Lett. 1990;64:2094-7.

87. Mori T, Sugiyama S, Byrne M, Johnson CH, Uchihashi T, Ando T. Revealing circadian mechanisms of integration and resilience by visualizing clock proteins working in real time. Nat Commun. 2018;9:3245.

88. Maity S, et al. VPS4 triggers constriction and cleavage of ESCRT-III helical filaments. Sci Adv. 2019;5:eaau7198.

89. Maity S, et al. Caught in the act: mechanistic insight into supramolecular polymerization-driven self-replication from real-time visualization. J Am Chem Soc. 2020;142:13709-17.

\section{Publisher's Note}

Springer Nature remains neutral with regard to jurisdictional claims in published maps and institutional affiliations.

Ready to submit your research? Choose BMC and benefit from:

- fast, convenient online submission

- thorough peer review by experienced researchers in your field

- rapid publication on acceptance

- support for research data, including large and complex data types

- gold Open Access which fosters wider collaboration and increased citations

- maximum visibility for your research: over $100 \mathrm{M}$ website views per year

At $\mathrm{BMC}$, research is always in progress.

Learn more biomedcentral.com/submissions 